\title{
A pragmatic strategy for implementing spatially correlated observation errors in an operational system: an application to Doppler radial winds
}

Article

Accepted Version

Simonin, D., Waller, J. A., Ballard, S. P., Dance, S. L. and Nichols, N. K. (2019) A pragmatic strategy for implementing spatially correlated observation errors in an operational system: an application to Doppler radial winds. Quarterly Journal of the Royal Meteorological Society, 145 (723). pp. 2772-2790. ISSN 1477-870X doi:

https://doi.org/10.1002/qj.3592 Available at https://centaur.reading.ac.uk/84168/

It is advisable to refer to the publisher's version if you intend to cite from the work. See Guidance on citing.

To link to this article DOI: http://dx.doi.org/10.1002/qj.3592

Publisher: Royal Meteorological Society

All outputs in CentAUR are protected by Intellectual Property Rights law, including copyright law. Copyright and IPR is retained by the creators or other copyright holders. Terms and conditions for use of this material are defined in the End User Agreement. 


\section{www.reading.ac.uk/centaur}

\section{CentAUR}

Central Archive at the University of Reading

Reading's research outputs online 


\title{
A pragmatic strategy for implementing spatially correlated observation errors in an operational system: an application to Doppler radial winds
}

\author{
D. Simonin ${ }^{\mathrm{a} *}$, J. A. Waller ${ }^{\mathrm{b}}$, S. P. Ballard ${ }^{\mathrm{a}}$, S. L. Dance ${ }^{\mathrm{b}}$, and N. K. Nichols ${ }^{\mathrm{b}}$ \\ ${ }^{\mathrm{a}}$ MetOffice@Reading, Meteorology Building, University of Reading, Reading, Berkshire, RG6 6BB, United Kingdom \\ ${ }^{\mathrm{b}}$ School of Mathematical, Physical and Computational Sciences, University of Reading, Reading, Berkshire, RG6 6BB, United \\ Kingdom
}

*Correspondence to: D. Simonin, MetOffice@ Reading, Meteorology Building, University of Reading, Reading, Berkshire, RG6 6BB, United Kingdom. E-mail: david.simonin@ metoffice.gov.uk

Recent research has shown that high resolution observations, such as Doppler radar radial winds, exhibit spatial correlations. High resolution observations are routinely assimilated into convection permitting numerical weather prediction models assuming their errors are uncorrelated. To avoid violating this assumption observation density is severely reduced. To improve the quantity of observations used and the impact that they have on the forecast requires the introduction of full, correlated, error statistics. Some operational centres have introduced satellite inter-channel observation error correlations and obtained improved analysis accuracy and forecast skill scores. Here we present a strategy for implementing spatially correlated observation errors in an operational system. We then provide the first demonstration of the practical feasibility of incorporating spatially correlated Doppler radial wind error statistics in the Met Office numerical weather prediction system.

Inclusion of correlated Doppler radial winds error statistics has little impact on the computation cost of the data assimilation system, even with a four-fold increase in the number of Doppler radial winds observations assimilated. Using the correlated observation error statistics with denser observations produces increments with shorter length scales than the control. Initial forecast trials show a neutral to positive impact on forecast skill overall, notably for quantitative precipitation forecasts. There is potential to improve forecast skill by optimising the use of Doppler radial winds and applying the technique to other observation types. 


\section{Introduction}

Error characteristics of atmospheric observations are complex and not straightforward to derive. Each meteorological instrument is accurate to within a given tolerance subject to its engineering specifications. This is called instrument error. However, in the context of data assimilation, there is a representation error that arises in addition to the instrument error. The sources of representation error include the variability of the observed field at scales different from those resolved by the assimilating dynamical model, observation pre-processing and/or the approximation of the observation operator (Janjić et al. 2017). Therefore, the total observation error can be expressed as the sum of the instrument error and a representation error. It is generally assumed that instrument error is uncorrelated and unbiased (any existing biases are assumed to have been removed). In contrast the error of representation is generally correlated and state dependent (Waller et al. 2014).

Idealized studies have shown that incorporating correlated observation errors in data assimilation systems leads to a more accurate analysis (Stewart et al. 2013; Stewart 2010; Healy and White 2005) and to the inclusion of more observation information content (Stewart et al. 2008), particularly on small scales (Rainwater et al. 2015; Fowler et al. 2018). Studies with operational data have shown that satellite inter-channel errors can exhibit significant correlations (Stewart et al. 2009, 2014; Bormann and Bauer 2010; Bormann et al. 2010; Waller et al. 2016a), and accounting for them in the assimilation results in improvements in the forecast skill score (Weston et al. 2014; Bormann et al. 2016; Campbell et al. 2017), but may affect the number of iterations required to solve the variational minimization problem (Tabeart et al. 2018). More recent research has shown that observation errors can also be spatially correlated (Waller et al. 2016c,a; Cordoba et al. 2017).

The UK public weather service has an emphasis on accurate forecasts/nowcasts of strong convective storms which can be responsible for major flooding events. In response, the UK Met Office has an operational convection permitting numerical weather prediction (NWP) system using a $1.5 \mathrm{~km}$ version of the Unified Model (UM) (Lean et al. 2008; Tang et al. 2013). Such a system requires the assimilation of new, high temporal and spatial resolution observations in order to provide an initial state that contains information at suitable scales (Gao and Stensrud 2012; Sun et al. 2014; Clark et al. 2015; Ballard et al. 2016). Such observations include, for example, mode-S aircraft data (e.g. de Haan and Stoffelen 2012; Strajnar et al. 2015; Lange and Janjić 2016), weather radar (e.g. Caya et al. 2005; Wattrelot et al. 2014; Wang and Wang 2017) or high resolution AMVs (e.g. Velden et al. 2017). However, due to the presence of correlated errors, there has been no attempt to operationally assimilate observations at a high spatial density. Instead, the observations are assumed to be spatially uncorrelated; the data is thinned to separation lengths where this assumption is understood to be reasonable and the error variances inflated to account for any neglected correlations (Buehner 2010). As a result, the quantity of high resolution observations, such as those provided by weather radar in the form of reflectivity, radial wind (Simonin et al. 2014) and refractivity, is severely reduced. This may result in a sub-optimal analysis and poorer forecasts. Therefore, in order to assimilate observations at a high spatial density the observation error correlations must be considered.

This work proposes a pragmatic strategy that allows the use of horizontally correlated observation errors. We describe the implementation of such a strategy within the Met Office operational variational assimilation scheme. Practical feasibility and possible impacts are demonstrated with NWP trial experiments using spatially correlated observation error for Doppler radial wind.

First, we present the current assimilation system used at the Met Office in Section 2. Subsequently, in Section 3, we describe in detail the implementation of the proposed strategy that allows use of correlated observation error statistics. After presenting the experimental details in section 4 , section 5 shows the impact of the new scheme when it is applied to Doppler radial wind observations for the assimilation system, the analysis and the forecasts. Finally we conclude in Section 6.

\section{The current Met Office approach}

In this section we describe the current Met Office variational data assimilation system software (VAR) and its parallelisation. We 
also describe the current treatment of observation error statistics in the assimilation.

\subsection{The data assimilation system}

In this section we describe some pertinent features of the current Met Office variational data assimilation (VAR) software (Lorenc et al. 2000; Rawlins et al. 2007). These schemes are based on the incremental approach of Courtier et al. (1994) and are applicable to 3D-Var and 4D-Var. Here we document them following the notation of Ide et al. (1997).

Given a full resolution non-linear forecast model, incremental variational assimilation seeks a simplified, perturbation model state increment $\delta w^{a} \in \mathbb{R}^{n_{s}}$ to a full resolution guess field $\mathbf{x}^{g} \in$ $\mathbb{R}^{n}$ such that the analysis at full resolution $\mathbf{x}^{a} \in \mathbb{R}^{n}$ at $t=T+0$ is given by

$$
\mathbf{x}^{a}=\mathbf{x}^{g}+\mathbf{S}^{-1} \delta \mathbf{w}^{a} .
$$

Here, $\mathbf{S}^{-1}$ is the incrementing operator; it is the generalised nonlinear inverse of a simplification operator $\mathbf{S}$ which reduces the full model's complexity and resolution to that of the perturbation (Ide et al. 1997). In the Met Office VAR schemes, where the full resolution non-linear model is the $\mathrm{UM}$, the operator $\mathbf{S}$ is also used to simplify multiple moisture and cloud variables to a single variable (Rawlins et al. 2007). We find the perturbation model state, $\delta \mathbf{w}^{a}$, at $t=T+0$ by minimizing a penalty function,

$$
\begin{aligned}
J(\delta \mathbf{w})= & \frac{1}{2}\left(\delta \mathbf{w}-\delta \mathbf{w}^{b}\right)^{T} \mathbf{B}^{-1}\left(\delta \mathbf{w}-\delta \mathbf{w}^{b}\right) \\
& +\frac{1}{2}\left(\mathbf{y}-\mathbf{y}^{o}\right)^{T} \mathbf{R}^{-1}\left(\mathbf{y}-\mathbf{y}^{o}\right) \\
= & J_{b}+J_{o},
\end{aligned}
$$

where $\delta \mathbf{w}=\mathbf{S}(\mathbf{x})-\mathbf{S}\left(\mathbf{x}^{g}\right)$ and $\delta \mathbf{w}^{b}=\mathbf{S}\left(\mathbf{x}^{b}\right)-\mathbf{S}\left(\mathbf{x}^{g}\right), \mathbf{x}^{b} \in \mathbb{R}^{n}$ is the background model state, $\mathbf{B} \in \mathbb{R}^{n \times n}$ is the background error covariance matrix and $\mathbf{R} \in \mathbb{R}^{p \times p}$ is the observation error covariance matrix. The penalty function minimizes the fit of the model state to the background state, $J_{b}$, and observations, $J_{o}$. Note that the variational problem is solved iteratively using a conjugate gradient method.

This work was conducted using a 3D-Var assimilation system with a centered window using first guess at appropriate time
(FGAT: Fisher and Andersson 2001; Lorenc and Rawlins 2005).

The observations $\mathbf{y}^{o} \in \mathbb{R}^{p}$ are distributed within an assimilation time window $\left[T-t_{\mathbf{w}}, T+t_{\mathbf{w}}\right]$. The background model state is 113 provided by a previous forecast and is interpolated in time to 114 the observation time. Following Lorenc and Jardak (2018), the 115 model prediction of the observations is given by $\mathbf{y}=H\left(\underline{\mathbf{G}} \mathbf{x}^{g}+{ }_{116}\right.$ $\left.\underline{\mathbf{G}} \mathbf{S}^{-1} \delta \mathbf{w}\right)$ where $\underline{\mathbf{G}}$ is the linear time- and space-interpolation of 117 the model generated field to the observation location and time and 118 $H$ is the non-linear observation operator.

In order to calculate the model prediction of the observations it is necessary to interpolate the primary variables of the forecast model and the perturbation forecast model to the observation locations. Therefore, for each observation we define:

- The array $C_{x}=\underline{\mathbf{G}}^{g}$ consisting of a vertical column of 124 the primary variables of the forecast model, interpolated 125 horizontally to the observation positions, valid at the 126 observation time.

- The array $C_{w}=\underline{\tilde{\mathbf{G}}} \mathbf{S}^{-1} \delta \mathbf{w}$ consisting of a column of 128 the primary variables of the perturbation forecast model, 129 interpolated horizontally (and in time for 4D-VAR) to 130 the observation positions. 3D-Var treats all increments at 131 the same analysis time (in the middle of the window) 132 so $\tilde{\mathbf{G}}$ incorporates a space-interpolation only; FGAT is 133 implemented by the time-interpolation to the exact time of 134 each observation, in $\mathbf{G}$.

- The array $\widehat{C}_{w}$, the derivative of the observation penalty 136 function $\left(J_{O}\right)$ with respect to the primary variables of the 137 perturbation forecast model $\left(C_{w}\right)$

\subsection{Parallelisation}

The current approach to the parallelisation of the VAR code 140 follows the Data Parallel paradigm (Pacheco 1997, section 2.2.3): all the processing elements (PEs) carry out the same operations 142 on different portions of the data set (figure 1 top panel). The data 143 is split into a number of geographical regions; this is known as 144 Domain Decomposition.

For VAR, the domain decomposition splits the $C_{w}$ columns 146 such that each PE has information containing all the vertical levels but only for a specified area of the horizontal-plane. The so s. ${ }^{\infty}$ 3 a

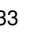
s.

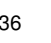
ar s. 
PE IDs assigned to each column of $C_{w}$ are stored in the vector $C w_{P E} \in \mathbb{R}^{p}$

As the observations are assumed to be independent and uncorrelated, they are simply spread across processors following the same regional decomposition as the model information (shown in figure 1). In this approach, the costs of the observation calculations are small compared to other components because there are no inherent message-passing or synchronisation delays This advantage outweighs the inefficient load-balancing, for the domains typically used. The allocated PE ID for each observation is stored in the vector $O b s_{P E} \in \mathbb{R}^{p}$. This strategy is applied to all observation types and to the model information such that $O b s_{P E}=C w_{P E}$

\subsection{Treatment of observation error statistics}

Observation errors are typically assumed to be temporally uncorrelated, and with no correlations between observation types, so that $\mathbf{R}$ is block-diagonal. This allows $J_{o}$ to be calculated independently for each observation type and hence reduces the cost of the matrix-vector products in equation (2). Also, for many observation types, it is assumed that the observation errors are independent, Gaussian white noise, so that the associated observation error covariance sub-matrix for a given observation in equation (2) is diagonal (no cross-correlation) and contains the sum of instrument and representation errors $\mathbf{R}=\mathbf{E}+\mathbf{F}$ (Lorenc et al. 2000). In this case the matrix-vector product simplifies to a series of scalar multiplications. There is one exception to this description. The current system accounts for correlated satellite inter-channel errors (Weston 2011; Weston et al. 2014). In this case, sets of observations with inter-channel error correlations provide information related to a single model column; hence the inclusion of correlated inter-channel error matrices is compatible with the current parallelisation strategy where observation and vertical model columns are distributed together between supercomputer processors (see Section 2.2 for a more detailed description). However, in the case of horizontally correlated observation error statistics, the existing data-structures do not allow the computation of the required matrix-vector products without excessive communication between processors.

\section{The new approach}

In this section we describe how the current Met Office variational 188 data assimilation system software (VAR) has been adapted to 189 exploit, and allow for, horizontal correlated observation error 190 statistics.

\subsection{Parallelisation}

As shown in section 2.2, the assimilation system is using the same domain decomposition for observations as model. However, in 194 order to make use of a full observation error covariance matrix, 195 $\mathbf{R}_{s}$, (i.e. variance and correlation), it is necessary to gather error- 196 correlated observations, and their model equivalent, on a single 197 processor as shown in the bottom panel of figure 1.

To accommodate full observation error covariance matrices, 199 the parallelization has been modified for observations that have 200 mutually correlated errors. These observations are assigned to a 201 family and sent to a single PE (figure 1 bottom panel) and are no 202 longer distributed on a PE according to its geographical location 203 but according to its family instead: $O b s_{P E} \neq C w_{P E}$. If no family 204 has been defined (observations with uncorrelated errors shown 205 as blue dots in the bottom panel of 1), then the distribution of 206 the information across the numerous processors is done in the 207 traditional way (i.e domain decomposition $O b s_{P E}=C w_{P E}$ ). 208

If some observations are believed to be correlated and 209 associated to families, the main steps of the algorithm are:

- Each family of observations is assigned to a unique 211 processor, following the $O b s_{P E}$ assignment.

- The $C_{w}$ 's are still distributed using the domain decomposi- $\quad 213$ tion (following the $C w_{P E}$ assignment), to allow horizontal 214 interpolation to be a local operation on each PE.

- At each iteration, all the $C_{w}$ 's associated with a family of 216 observations are gathered into the processor assigned to this 217 family.

- The observation penalty $\left(J_{O}\right)$ is calculated.

- The last step is to redistribute the $\widehat{C}_{w}$ 's to their original 220 location according to the $C w_{P E}$ assignment.

This new approach could significantly increase the communi- 222 cation between processors. However, the added communications 223 are not all-to-all; a set of lookup tables have been implemented 224 
to ensure a "link" between $O b s_{P E}$ and $C w_{P E}$. This restricts the communication to a minimum. In addition, the dissociation between the $O b s_{P E}$ and $C w_{P E}$ offers the opportunity to improve the load balancing. Observations are rarely uniformly distributed across the model domain, which implies that some processors will have more work than others if a domain decomposition is used With this new approach, families can be allocated to the least loaded processor and improve the overall load balancing of the system. The only real limitation of this approach is in the definition of families. For observation types such as radar observations, or GPS, where natural groupings exist, it is relatively easy to use. However for observations such as geostationary satellite imagery, where the entire model domain is covered by one single image, the creation of families is more difficult. One approach for this case is for families to represent a section of the domain, with extra observations forming a halo.

\subsection{Treatment of observation error statistics}

The proposed approach for using spatially correlated errors is to treat each family in a similar way to the current approach for inter-channel correlations mentioned in section 2: Since $\mathbf{R}$ and its inverse change each assimilation due to the quality control process and observation availability, a Cholesky decomposition method is used to calculate the observation penalty, $J_{O}$ as described in Weston et al. (2014) . This avoids the need to compute the inverse observation error covariance matrix directly. The method requires positive definite symmetric matrices, which covariance and correlation matrices are by definition, and is computationally cheaper than alternatives such as Gaussian elimination. This approach for handling correlated observation errors relies on the full $\mathbf{R}$ being block diagonal, otherwise it may be necessary to use an approximation method such as Yaremchuk et al. (2018).

For each family it is necessary to determine the full spatial observation error correlation matrix $\mathbf{C}$ and a matrix of standard deviations D. For families containing fixed observations (observations at the same locations at each assimilation step) it may be possible to store a single fixed observation error covariance matrix. However, as mentioned earlier, due to quality control procedures and the intermittent nature of most observations, the observation error covariance matrix for each family will change at each assimilation step. It therefore makes sense to derive $\mathbf{C}$ dynamically by simply providing a correlation function and a pre-derived correlation length scale for each type 266 of family. For example $\mathbf{C}$ may be derived using,

$$
\mathbf{C}_{i, j}=e^{\left(\frac{-\left|\Delta y_{i, j}\right|}{L_{r}}\right)} .
$$

where for a given family, $\Delta y_{i, j}$ is the separation distance between

a pair of observations $\mathbf{y}_{i}$ and $\mathbf{y}_{j}$ and $L_{r}$ is the correlation 269 lengthscale. Similarly $\mathbf{D}$ is constructed using pre-derived variance 270 for each family.

After determining the full spatial observation error correlation matrix and matrix of standard deviations, the observation error 273 covariance matrix $\mathbf{R}_{f}=\mathbf{D C D}$ and the observation penalty $\left(J_{o}\right) \quad 274$ can be calculated as follows:

1. Calculate a vector of observation minus model equivalent 276 differences $d_{o}^{b}=\left(\mathbf{y}^{o}-\mathcal{H}(\mathbf{x})\right)$.

2. Calculate the sensitivity $\mathbf{q}=\mathbf{R}_{f}^{-1}\left(\mathbf{y}^{o}-\mathcal{H}(\mathbf{x})\right)$ using a 278 Cholesky decomposition (Golub and Van Loan 1996). 279 The Cholesky decomposition avoids the need to invert 280 the observation error matrix. Instead the sensitivity is 281 calculated by first factorizing $\mathbf{R}_{f}=\mathbf{U} \mathbf{U}^{T}$, where $\mathbf{U}$ is an 282 upper triangular matrix, then solving for $\mathbf{q}$ using forward, $\quad 283$ and backward substitution.

3. The total observation penalty $J_{O}$ for the family is calculated 285 by multiplying the sensitivity by the observation minus 286 model equivalent differences,

$$
J_{o}=\frac{1}{2}(\mathbf{y}-\mathcal{H}(\mathbf{x}))^{T} \mathbf{R}_{f}^{-1}(\mathbf{y}-\mathcal{H}(\mathbf{x})) .
$$

The gradient of $J_{o}$ needed for the variational minimisation is calculated using,

$$
\frac{\partial J_{o}}{\partial \mathbf{x}}=\mathbf{H}^{T} \mathbf{R}_{f}^{-1}\left(\mathbf{y}^{o}-H(\mathbf{x})\right) .
$$

\section{Experimental details}

The model used in this study is the operational UKV model. It is 291 a variable-resolution version of the nonhydrostatic UM (Davies 292 et al. 2005), allowing an explicit representation of convective 293 
processes as described in Lean et al. (2008). The horizontal grid has a $1.5-\mathrm{km}$ fixed resolution on the interior surrounded by a variable-resolution grid that increases smoothly in size to $4 \mathrm{~km}$ and has 70 vertical levels. The variable-resolution grid allows the downscaled boundary conditions, taken from the global model, to spin up before reaching the fixed interior grid. The initial conditions are provided from the operational 3 hourly $3 \mathrm{D}$ variational assimilation scheme that uses an incremental approach (Courtier et al. 1994) and is a limited-area version of the Met Office variational data assimilation scheme (Lorenc et al. 2000; Rawlins et al. 2007). The assimilation uses a vertical adaptive mesh that allows the accurate representation of boundary layer structures (Piccolo and Cullen 2011, 2012).

The background error covariance has been derived using the Covariances and VAR Transforms software (Wlasak and Cullen 2014), which is Met Office covariance calibration and diagnostic tool that analyses training data representing forecast errors using the National Meteorological Center (NMC) lagged forecast technique or ensemble perturbations. Here an NMC method has been applied to $(T+6 h)-(T+3 h)$ forecast differences to diagnose variances and correlation length scales.

For this study, we are using a 3DVar analysis system with first guess at appropriate time (FGAT). The background field is provided by a $T+3$ forecast (actually time interpolated to observation time using fields every 30 minutes in the $3 h$ observation window for FGAT). In addition to Doppler radial winds used at the centre of the assimilation window, the analysis uses hourly surface synoptic observations of temperature, wind, pressure, humidity and visibility, hourly wind profiler data, hourly satellite radiances, satellite winds and aircraft data, radiosonde and hourly GPS water vapour paths (note that hourly referes to the frequency usage of the observation) . Radar-derived surface precipitation rates available every $15 \mathrm{~min}$ are included via latent heat nudging from $\mathrm{T}-0.5 \mathrm{~h}$ to $\mathrm{T}+0.5 \mathrm{~h}$ and hourly cloud-coverderived 3D relative humidity profiles via moisture nudging (Jones and Macpherson 1997; Dixon et al. 2009). The nudging was done over a period surrounding the analysis time, in addition to incremental analysis updating of the 3D-Var analysis increments.

The Doppler radial winds are provided by 18 Doppler Weather radars spread over the United Kingdom. Each radar produces 5 plan position indicator (PPI) scans every 10 minutes. The Doppler

radial winds are assimilated using a simple observation operator 335 where the horizontal model background winds are projected onto 336 the slant of the radar beam (vertical motion is ignored) (Simonin 337 et al. 2014). To reduce the density of the observations, multiple 338 observations are made into a single super-observation $\left(3^{\circ}\right.$ by $\left.3 \mathrm{~km}\right) \quad 339$ and then thinned using Poisson disk sampling, as described in 340 Simonin et al. (2014).

The observation error correlation matrices are calculated 342 dynamically as described in section 3 . In the correlation matrices 343 we only consider horizontal correlations; we neglect vertical 344 correlations as there are unlikely to be multiple observations, 345 and hence vertically correlated errors, in a single model column. 346 Instead we assume that observation errors are correlated only if 347 the observations are within a height band as described in Waller 348 et al. (2016c). This assumption results in a sparse block diagonal 349 observation error correlation matrix. Using this approach the 350 number of observations in a family cannot exceed 2000. When 351 Doppler radial wind observation errors are assumed uncorrelated, 352 the standard deviations for the control experiment are based on 353 those described in Simonin et al. (2014) and evolve with range, 354 whereas when correlation is accounted for, the standard deviations 355 and length scales $L_{r}$ are based on those calculated in Waller et al. 356 (2016c). A comparison of the variances from both observation 357 error matrices $(\mathbf{R})$ is shown in figure 2 as a function of height 358 for the $1^{\circ}, 2^{o}$ and $4^{o}$ radar beams. The length scales $L_{r}$ have been $\quad 359$ determined by fitting Markov functions (eq. 3) to the estimated 360 horizontal correlations. We note that the length scales $L_{r}$ are 361 dependent on both the height of the observation and the radar 362 beam elevation. Neither the prescribed variances nor length scales differ between radars. However, due to the intermittent nature of the observations, the observation error covariance matrices do 365 differ between radars; similarly, for any given radar the error 366 covariance matrices differ at each assimilation time.

The impact of including horizontally correlated Doppler radial 368 wind errors was investigated by running three experiments using 369 data for the period 1-20 May 2016. As shown in table 1, the 370 Control experiment uses a diagonal observation error matrix, 371 whereas both experiments Corr-R-3km and Corr-R-6km use a 372 
correlated observation error matrix. The Control run and the Corr-

R-6km experiment use a $6 \mathrm{~km}$ thinning distance whereas the Corr-

$\mathrm{R}-3 \mathrm{~km}$ experiment uses a $3 \mathrm{~km}$ thinning distance. We note that the Control run and the Corr-R-6km experiment use the same set of observations; therefore, comparisons between these experiments allow us to determine the impact of including spatially correlated observation errors in the system. Comparisons between the CorrR-6km and Corr-R-3km experiments allow us to assess the impact of including denser observations (permitted by the inclusion of the correlated errors). Results from an additional experiment using the control's set-up with $3 \mathrm{~km}$ thinning instead of $6 \mathrm{~km}$ will be presented periodically to add context. This experiment (Diag-R$3 \mathrm{~km}$ ) is known to be sub-optimal with the analysis degraded compared to the control. Comparison to the other experiments will positively bias the impact of correlated observation error; therefore, the authors limit the discussion of this experiment in the manuscript.

\section{Initial Results}

The initial impact of including the correlated observation error when assimilating Doppler radial wind has been assessed in three ways. First, we consider the computational performance of the system and its operational viability. Then we consider the relative impact on analysis and innovation accuracy by considering observation-minus-analysis and observation-minus-background statistics. Finally general forecast performance and specific quantitative precipitation forecast verification are presented.

\subsection{Variational data assimilation system performance}

This section focuses on the performance of the variational data assimilation system (VAR) during the trial.

Both the Control and Corr-R-6km experiments used a thinning distance of $6 \mathrm{~km}$, which yield an average of 2000 Doppler radial wind observations per cycle. The Corr-R-3km experiment, however, use a reduced thinning distance of $3 \mathrm{~km}$, which provides on average four times more (8000) Doppler radial wind observations per assimilation cycle.

Table 2 shows the average iteration and evaluation count for each experiment. The iteration and evaluation count from each run are very similar. (Note that one evaluation is one calculation of the penalty function, and one iteration is equivalent to one cycle of the minimisation algorithm). This result is most significant when considering that the Corr-R-3km experiment used four 413 times more Doppler radial wind observations. When comparing 414 the mean iteration/evaluation count to the standard deviation we 415 find that for all experiments there are substantial differences 416 observed between different assimilations. The large variance is 417 expected since we are using a regional data assimilation system 418 where the total number of observations can change significantly 419 depending on the time of assimilation (e.g. day vs. night). We note 420 that when comparing timeseries of iteration/evaluation counts there are minimal differences between the three experiments (not shown) and all follow a diurnal cycle.

Table 2 also shows the average and standard deviation of the of observation and background penalty values $\left(J_{o}\right.$ and $J_{b}{ }_{425}$ respectively). The changes in the mean value of $J_{o}$ and $J_{b}{ }_{426}$ suggest that the overall observation weight is reduced and more 427 importance is given to the background information as shown from 428 theoretical studies by Seaman (1977) or Stewart et al. (2008). 429 This is evident when Corr-R- $6 \mathrm{~km}$ is compared to the Control as 430 both experiments use the same observation count. Corr-R-6km 431 has an increased (reduced) observation (background) penalty. As 432 values of $J_{o}$ and $J_{b}$ are affected by the observation count, Corr-R- 433 $3 \mathrm{~km}$ needs to be compared to a Control experiment using $3 \mathrm{~km} \quad 434$ thinning (Diag-R-3km ). The comparison of Corr-R-3km with 435 Diag-R-3km gives similar results to the Corr-R-6km/Control 436 comparison. The mean values of $J_{o}$ and $J_{b}$ for Diag-R-3km are 437 equal to 9679.28 and 2277.59 respectively, whereas for Corr-R- 438 $3 \mathrm{~km}$ these values are equal to 10134.63 and 2050.53 . The decrease $\quad 439$ in background penalty between Diag-R-3km and Corr-R-3km 440 more or less matches the increase in observation penalty between 441 the two experiments.

Table 3 shows the performance of the assimilation over 443 the trial period, as well as over 10 iterations, for the three 444 experiments. Comparing the experiments we see that the increased 445 communication did not impact on the performance of the code. 446 The cost of computing $J_{O}$ is minimal compared to that of $J$ as $\quad 447$ wells as the wall-clock time. The cost of $J_{O}$ remains minimal and 448 there is little change in the total cost of $J$ even when correlated 449 observation error are used and observation count is increased. 11 ,

\section{,} 420

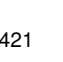
. . s.

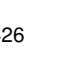
. . . . , . , "

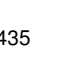

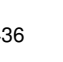
. 
Overall results show that the proposed strategy to introduce correlated observation error statistics does not diminish the computational performance of the assimilation system. Furthermore, denser observations with correlated errors can be assimilated without increasing the computational cost.

\subsection{Impact on the analysis}

Residual $(O-A)$ and innovation $(O-B)$ statistics provide a quantitative measurement of the impact of the correlated observation error upon the analysis for individual observation types. The $O-A$ are retrieved from the assimilation system as the residual at the end of the minimisation. First, we note that the mean bias from the innovation or the residual for this Doppler radial wind will always tend toward zero for a large quantity of observations due to the radial nature of the observation (Salonen et al. 2007). Instead figure 3 shows the $O-B$ and $O-A$ standard deviation ( $\sigma_{O-B}$ and $\sigma_{O-A}$ hereafter) from the Control, Corr-R$6 \mathrm{~km}$ and Corr-R-3km as a timeseries for each cycle over the of the trial. The time series of Doppler radial wind's $\sigma_{O-B}$, yield similar results, with mean $\sigma_{O-B}$ over the length of the trial for the Control, Corr-R-6km and Corr-R-3km is equal to $2.77,2.76$ and 2.73 respectively. Here the background is a $T+3$ forecast from the previous cycle ( 3 hourly data assimilation system). More pronounced differences between the control and the experiments are visible in the Doppler radial wind's $\sigma_{O-A}$ (figure 3). In the case of Corr-R-6km (figure 3-a), the values of $\sigma_{O-A}$ are consistently slightly higher than those for the Control. In the case of Corr-R-3km (figure 3-b) the $\sigma_{O-A}$ are comparable to the values yielded by the Control.

The differences in $\sigma_{O-A}$ between the two runs with identical observation count (i.e. Control and Corr-R-6km) confirm the results of the previous section. Despite the fact that the observation error matrix used in Corr-R-6km had smaller or equivalent variance compared to those prescribed for the Control experiment (figure 2), the weight of the Doppler radial wind observations was reduced in Corr-R-6km. This in turn reduces the fit to the observations and increases analysis error. This increase in analysis error is not seen in the Corr-R-3km's experiment where the $\sigma_{O-A}$ shows similar values compared to the Control. The reduction in the observation weighting has been reversed by the increased observation count. This is supported by considering the additional

Diag-R-3km experiment, where $\sigma_{O-A}$ is consistently lower 491 (mean value of 1.20) compared to the Control (mean value of 492 $1.57)$.

Before considering the impact on other observation types, 494 we first consider how the structured wind increments may have been modified by the introduction of correlated observation error.

Figure 4 shows the mean length scale, the mean variance and 497 maximum values of the zonal wind increment at each model level 498 over the trial. Length scale is simply defined as the fourth root 499 of the ratio of the variance of a field $(\phi)$ and the variance of 500 its Laplacian (calculated using a second-order finite difference 501 approximation) (Descombes et al. 2015); that is

$$
\mathbf{L}=\left(\frac{8 \cdot \operatorname{variance}(\phi)}{\operatorname{variance}\left(\nabla^{2} \phi\right)}\right)^{1 / 4}
$$

We note that the mean increment can be related to systematic 503 error in either observations or the model (Rodwell and Palmer 504 2007). However it has been shown that the Doppler radial wind 505 observations used here are unbiased (Simonin et al. 2014). When 506 the correlated observation error covariance matrix is introduced 507 (Corr-R-6km) the zonal wind increment becomes smoother 508 with smaller extremes at all model levels. The introduction of 509 correlation acts as a low-pass filter, reducing the weight from 510 individual observations and increasing the importance of the 511 background information. This is consistent with the results from 512 the $\sigma_{O-A}$. However, increasing the observation density (Corr-R- 513 $3 \mathrm{~km}$ ) counterbalances the effect of the correlated $\mathbf{R}$, by increasing 514 the amplitude and the variance of the increment values at all 515 levels so that the values are closer to the Control experiment. It 516 produces increments with smaller length scales than the Control 517 from the assimilation of denser observations which are more able 518 to represent smaller scale features.

We now consider the impact from the introduction of the 520 correlated observation error covariance matrix for the Doppler 521 radial wind on the fit to other observations assimilated during 522 the trial. Figure 5 shows the trial average ratio of $\sigma_{O-A}$ and 523 $\sigma_{O-B}$, between the experiments and the Control for all the wind 524 observation types used in the trial. The error bars shown in 525 figure 5 and subsequent figures, represent the $95 \%$ confidence 526 
level. Due to high statistical variability between cycles, one should only consider the significant values to assess the impact. Most trial average innovation and residual standard deviations from the Corr-R-6km and Corr-R-3km yield smaller values compared to the Control, with Corr-R-3km outperforming Corr-R-6km. This trend is not completely homogeneous with, for example, mixed impact for the scatterometer wind (not statistically significant). Although not significant, the $O-A$ and $O-B$ from Corr-R$6 \mathrm{~km}$, exhibit a degradation for meridional wind from Sonde and Aircraft respectively. Note that Sonde $\mathrm{U}$ and $\mathrm{V}$ account for radiosonde profiles as well as wind-profiler observations. For all wind observations, the additional Diag-R-3km produces innovation and residual values (figure 5) that never improve upon the results of either the Corr-R-6km or Corr-R-3km. Furthermore, the innovation and residual values are significantly worse compared to the Control, with a decrease in analysis and background accuracy reaching $1 \%$ and more on a few occasions. For example, compared to the Control, $\mathrm{U}$ and $\mathrm{V}$ wind from Sonde are degraded by $2 \%$ in the $\sigma_{O-A}$ and at least $0.5 \%$ in the $\sigma_{O-B}$. Note that Corr-R-3km shows an improvement of $0.5 \%$ in the $\sigma_{O-A}$ and at least $0.75 \%$ in the $\sigma_{O-B}$.

The reduction in analysis error and improved innovations are equally visible when considering the results from satellite observations (figure 6). Again the general impact is stronger for Corr-R-3km. For the rest of the surface and upper-air observations (figure 7), the impact seen when considering Corr-R-6km is very much neutral, whereas Corr-R-3km still shows benefit. The statistics for relative humidity and potential temperature observations from sonde are neutral to negative in the $\sigma_{O-A}(7-\mathrm{a})$, but improve in the $\sigma_{O-B}(7-\mathrm{a})$. Again the additional Diag-R-3km (not shown) produces residual values that are worse compared to the Control with a maximum increase of $3 \%$ for relative humidity. Also, the $\sigma_{O-B}$ do not outperform the Corr-R-6km or Corr-R$3 \mathrm{~km}$ experiments. For satellite observations, Diag-R-3km has small overall improvement in comparison to the Control $(0.3 \%$ in $\left.\sigma_{O-B}\right)$. However, Corr-R-6km or Corr-R-3km experiments are still significantly superior.

We now summarize the results from this section. From the analysis of $O-B$ and $O-A$ it is clear that the introduction of correlated observation error for the Doppler radial winds had a general benefit in reducing the analysis error. From the results of the residual statistics $\left(\sigma_{O-A}\right)$ and the shape of the wind 568 increments, we see that the introduction of correlated observation error has a multi faceted effect (Daley 1991). The changes 570 in the $\sigma_{O-A}$ from Corr-R-6km experiment compared to the 571 Control, as well as the observation and background penalty values, $\quad 572$ demonstrated that the Control experiment settings were producing 573 an analysis that was over-fitting the Doppler radial wind. The 574 use of a diagonal observation-error covariance matrix when 575 observation errors are clearly horizontally correlated (Waller et al. $\quad 576$ 2016c) produced a suboptimal analysis (Liu and Rabier 2003). 577

When the observation errors are correlated with a length scale 578 of $20-30 \mathrm{~km}$ (Waller et al. 2016c), thinning the data to a $6 \mathrm{~km} 579$ spacing does not result in negligible error correlations between 580 assimilated observations. By introducing correlated observation 581 error statistics in the assimilation algorithm (Corr-R-6km), the 582 assimilation algorithm acts like a low-pass filter on the observation $\quad 583$ increments. Reducing the thinning distance shows benefit only 584 when the correlation in the observation errors are accounted 585 for as demonstrated by results from Corr-R-3km. Omitting 586 the correlation when using a dense network of observations, 587 only produces a sub-optimal system, where dense observations are over-fitted and the general analysis error is increased. By contrast accounting for correlation when using a dense network of observations, increases the potential number of neighbour observations $y_{j}$ to an observation $y_{i}$, allowing for synergy 592 between more pairs of observations, as described by Fowler et al. 593 (2018), as well as allowing the information content from smaller 594 scales to be exploited. This transforms the assimilation algorithm 595 and allows it to behave more like a high pass filter compared to 596 the Corr-R-6km setting.

We support these results using simple model experiments 598 (details are given in the appendix). We designed three experiments 599 to imitate the changes in observation density between the Control, 600 Corr-R-6km and Corr-R-3km experiments. Figure 8 shows the 601 eigenvalues of the analysis error covariance matrix in observation 602 space for the three simple model experiments:

- The Control experiment is qualitatively similar to the 604 simple model experiment shown as a black curve in 605 
figure 8 . Here the simple model is using a diagonal observation error covariance but the true observation error covariance contains some correlation and the state is half observed.

- The Corr-R-6km experiment has a similar character to the simple model experiment shown as a gray curve in figure 8 , where a correlated observation error covariance is used and the state is half observed.

- The Corr-R-3km experiment is qualitatively similar to the simple model experiment shown as a black dashed curve in figure 8. A correlated observation error covariance is used and the state is fully observed.

Figure 8 shows that assimilating observations with the correct error statistics reduces the analysis uncertainty at all scales compared to the case when the observation error correlations are neglected. However, in the case where the observation density is coarse, most of the reduction in analysis uncertainty is seen at large scale (grey curve of figure 8). Increasing the observation sampling when correlated observation errors are used, further reduces the analysis uncertainty. However, this time the additional reduction in uncertainty takes effect at small scales (dashed curve of figure 8), which is consistent with the analysis of our experiments.

\subsection{Forecast performance}

This section focuses on the impact on the forecast from the introduction of Doppler radial wind's correlated observation error.

\subsubsection{Overall forecast performance}

In order to quantify forecast skill of a variable such as temperature, wind or cloud cover it is possible to check the root mean square (RMS) or the equitable threat score (ETS) difference (Ebert et al. 2003) between an observed quantity and its forecast equivalent at a range of lead times, from $\mathrm{T}+6$ to $\mathrm{T}+36$ at 6 -hour intervals. The forecast value at observation locations is calculated from a simple bilinear interpolation of the forecast taking a distance-weighted average of the four surrounding grid point values. From the values derived following the above process an index that summarizes this skill score can be determined so as to make it easier to tell how a given trial experiment is performing with respect to the Control.
The Met Office's UK NWP Index is defined as a weighted 644 average of $\mathrm{T}+6$ to $\mathrm{T}+36$ skill scores over the UK domain, for $1.5 \mathrm{~m} \quad 645$ temperature, $10 \mathrm{~m}$ wind (speed and direction), precipitation (equal 646 to or greater than $0.5,1.0$ and $4.0 \mathrm{~mm}$ over the preceding 6 hours), $\quad 647$ total cloud amount (equal to or greater than 2.5, 4.5 and 6.5 oktas), $\quad 648$ cloud base height (given at least 2.5 oktas and equal to or less than 649 100,500 and $1000 \mathrm{~m}$ above ground) and near-surface visibility 650 (equal to or less than 200, 1000 and $4000 \mathrm{~m}$ ).

Table 4 shows the results of the surface verification as 652 percentage of improvements. For the Corr-R-6km UK NWP 653 index changes by $-0.005 \%$ compared to the Control run. For 654 the Corr-R-3km UK NWP index changes by $+0.021 \%$ compared 655 to the Control run. Neither trial presents statistically significant 656 differences in skill with respect to the Control run.

\subsubsection{Impact on precipitation}

So far we have concentrated our effort on the validation of 659 the forecast performance overall. However, one of the main 660 motivations of convective-scale assimilation is to improve short- 661 term quantitative precipitation forecasts (QPF). Verification 662 methods have conventionally been designed to assess the model 663 forecast at point locations only. However, the temporal and 664 spatial intermittent nature of a parameter such as rain makes 665 these approaches unsuitable in general (Droegmeier 1997). This 666 problem is amplified in this study because the $1.5 \mathrm{~km}$ model 667 resolution is high enough to represent small-scale features and 668 local variability. In response to this problem, a growing list of 669 methods to verify precipitation forecasts based on the physical 670 realism or spatial closeness to observations have been developed 671 (Gilleland et al. 2009). Some techniques have concentrated on 672 object verification (Ebert and MacBride 2000; Davis et al. 2006; 673 Johnson and Wang 2013) by classifying rain features according to 674 their characteristics. Other methods have focused on the spatial 675 error and one such score is the Fractions Skill Score (FSS) 676 introduced by Roberts and Lean (2008). The FSS provides a 677 measure of the spatial agreement between two fields by comparing 678 the fractional differences in the coverage of rain over differing 679 sized squares (neighborhoods) centered at every grid square. More 680 about the definition and use of the FSS can be found in the 681 original paper by Roberts and Lean (2008) and then subsequently 682 
in Roberts (2008), Mittermaier and Roberts (2010) or Skok

(2015). Here the two fields of hourly accumulations of surface precipitation are from the forecast itself and the radar composite of derived rain rate.

Figure 9 shows the difference in fraction skill score between the experiments (Corr-R-6km: figure 9-a; Corr-R-3km: figure 9b) and the Control as a Hinton diagram for different forecast lead times and different thresholds of hourly rainfall accumulation. The sign and the amplitude of the change in FSS values $(\triangle F S S)$ are shown with the color and size of the square respectively: positive values (positive impact) are shown as grey squares, whereas negative values (negative impact) are shown as black squares. The introduction of the correlated observation error only (CorrR-6km) does not show any real impact on precipitation (figure 9-a). The $\Delta F S S$ values are small $(\max |\Delta F S S|=0.009)$ with an almost homogeneous distribution of positive and negative impact. The results are more promising when the correlated observation error is introduced in association with an increase in observation density (figure 9-b). The $\triangle F S S$ values are larger compared to the previous comparison, but more importantly, a positive impact can be seem until $T+7$ forecast lead time. The biggest positive impact is found for low threshold values (e.g. $0.2 m m h^{-1}$ and $90^{\text {th }}$ percentile).

Note that the FSS values for the Control forecasts, for a neighbourhood size of 41 grid boxes, were all well above 0.6 indicating an already skillful forecast; although little impact can be seen over the entire period of the fully cycled trial, individual cycles do show some improvements. Figure 10 gives an example of the sort of differences that can be seen and shows an hourly accumulated precipitation $T+3$ forecast valid at 1500 UTC on the 7th of April 2016, for Control, Corr-R-6km, and Corr-R-3km. During the 7th of April 2016, a band of showers developed and moved southwards, producing heavy precipitation on the east and central part of the UK. Compared to the observed radar derived hourly rain accumulation (figure 10-a), the Control (figure 10-b) produced showers that were typically too sparse and locally far too intense. The Corr-R-6km improved the shower coverage, but the real benefit of including correlated observation error is visible in the Corr-R-3km experiment (figure 10-d), where shower coverage and intensity was noticeably improved. This is supported by the

FSS value shown in figure 10-e and $\mathrm{f}$.

The improvement seen in this particular forecast can be attributed to the change in observation weight. When accounting for correlated observation error the observation uncertainty information is no longer mutually independent. This results in a small down-weighting of the observations as demonstrated by the Corr-R-6km experiment (Figure 4). This effect results in a small benefit to the forecast and FSS (Figure 10c and e) as the Control experiment was over-fitting the Doppler radial wind producing broad analysis increments (Figure 4). Increasing the observation density in conjunction with correlated observation errors negates the smoothing effect seen in Corr-R-6km. The use of more accurate error statistics enables an improved representation of the small scale information content from the observation resulting in a more balanced analysis increment (Figures 4 and 8). Over time the small scale information propagates through the system and produces improved forecasts as seen in Figures 10d.

In this work, we provide a pragmatic strategy that allows 741 the use of correlated observation errors in a high dimensional 742 data assimilation system. We describe the implementation of 743 this strategy in the Met Office system and then present a study demonstrating the practical feasibility of including horizontally correlated Doppler radial wind observation error statistics and 746 its impact using an operational NWP system. The new strategy was achieved by altering the usual Data Parallel paradigm; rather 748 than distributing observations with correlated errors using a 749 domain decomposition, the observations are instead distributed 750 in families that have mutually correlated errors as described in 751 section 3.1. The second significant change relates to the actual 752 use of the horizontally correlated observation errors statistics in 753 the derivation of the observation penalty. This was implemented 754 following the description presented in section 3.2.

A trial has been run for 20 days using the Met-Office UKV 756 model configuration and 3D-Var, including a Control experiment 757 with the operational settings (diagonal $\mathbf{R}$ ), an experiment using 
covariance matrix, and an experiment using correlated observation

error statistics with increased observation density.

Analysis of the impacts from the introduction of Doppler radar radial wind horizontal correlated observation errors on the data assimilation system and forecast skill have also been presented. The introduction of correlated observation error has changed the response from the variational system as follows:

- Introducing horizontal correlated observation errors improves the $O-A$ and $O-B$ statistics in both experiments. This suggests that the Control settings with a diagonal observation error covariance matrix causes the assimilation to over-fit Doppler radial observations.

- The positive impact of the introduction of correlated observation error is stronger on the $O-A$ and $O-B$ statistics when dense observations are used. We showed that introducing the correlated observation error, whilst keeping the observation density fixed, has little impact on the analysis uncertainty at the small scales. However this is remedied by increasing the observation density that introduces additional observation information at small scales.

- The inclusion of correlated observation error statistics allows dense observations to be assimilated without detriment to the analysis quality.

- We showed that by accounting for the correlation in the Doppler radial wind observation error, observation density can be increased without any degradation to the computational speed of the assimilation system.

Our results suggest that the use of a diagonal $\mathbf{R}$ (Control experiment) created a suboptimal system, where a $6 \mathrm{~km}$ observation thinning distance was too dense (e.g. Liu and Rabier (2003) or Stewart et al. (2008)). As shown for example by Daley (1991) or Fowler et al. (2018) the system's responses to correlated observation error are complex and make use of observation information at specific scales. However, we showed that the general behavior of the data assimilation system is comparable to what can be expected using a simple model.

The impact on the forecast is more subtle. A small positive signal can be seen when the observations are compared to the model background within the assimilation system. This indicates

that the impact on the forecast lasts long enough to improve the 800 model background and consequently benefit the assimilation in 801 a cycling NWP system. Regarding the conventional verification 802 scores, the results indicate that over the length of the forecast there 803 is a small positive impact, if any. A stronger signal is visible in 804 the QPF scores. A positive impact can be seem until a forecast 805 lead time of $T+7$. The biggest positive impact is found at low 806 threshold values, which implies an improvement in the location of 807 the rain. For all verification scores, the increase in the observation 808 density yields better results.

To the best of our knowledge this is the first operational 810 implementation of horizontal correlation observation errors in 811 a data assimilation system for numerical weather prediction. 812 Despite a marginal impact on the forecast, the introduction 813 of the correlated observation error allows the assimilation to 814 make better use of the observations by allowing the assimilation 815 of very dense observation networks, such as radar, without 816 any cost (no significant increase of wall clock time) to the 817 assimilation. We note that we have only considered the impact 818 for a single case study (20 days). Furthermore, the only alteration 819 in the experiments has been the inclusion of the correlated 820 observation errors. Further studies are required to analyse the 821 impact for different meteorological conditions. Improved settings 822 for operational parameters associated with Doppler radial wind 823 assimilation may also benefit the forecast. This may include 824 testing for statistical consistency of background and observation 825 errors using the diagnostic of (Desroziers et al. 2005). In addition, $\quad 826$ since this work, the Met-Office operational system for convective $\quad 827$ scale numerical weather prediction system has been upgraded to 828 4D-VAR. Therefore this system is now being extended to the 4D- 829 VAR framework

Here we present a simple example to help explain the results given 832 in Section 5.

In statistical linear estimation theory, the analysed state, $x^{a}$, is 834 given by

$$
\mathbf{x}^{a}=\mathbf{x}^{b}+\delta \mathbf{x}^{a}=x^{b}+\widetilde{\mathbf{K}} \mathbf{d}_{b}^{o},
$$


where $\delta \mathbf{x}^{a}$ is the analysis increment, $x^{b}$ is the background state, $\mathbf{d}_{b}^{o}$ the innovation vector,

$$
\mathbf{d}_{b}^{o}=y^{o}-\mathbf{H} x_{b},
$$

and $\widetilde{\mathbf{K}}$ the gain matrix,

$$
\widetilde{\mathbf{K}}=\widetilde{\mathbf{B}} \mathbf{H}^{T}\left(\mathbf{H} \widetilde{\mathbf{B}} \mathbf{H}^{T}+\widetilde{\mathbf{R}}\right)^{-1} .
$$

The matrices $\widetilde{\mathbf{B}}$ and $\widetilde{\mathbf{R}}$ are the assumed background and observation error covariance matrix respectively.

To understand the impact on the analysis of using a sub-optimal observation error correlation matrix we consider the analysis error covariance matrix, A. If we know the exact background error statistics, $\widetilde{\mathbf{B}}=\mathbf{B}$, but are knowingly using an incorrect observation error covariance matrix, $\widetilde{\mathbf{R}} \neq \mathbf{R}$ then the analysis error covariance matrix is given by,

$$
\mathbf{A}=(\mathbf{I}-\widetilde{\mathbf{K}} \mathbf{H}) \mathbf{B}+\widetilde{\mathbf{K}}(\mathbf{R}-\widetilde{\mathbf{R}}) \widetilde{\mathbf{K}}^{T} .
$$

We consider the impact on the analysis error covariance using three simple model experiments. We assume that our background is evaluated on 128 equally spaced points in a 1D periodic domain, $(-32 \pi, 32 \pi]$. In order to compare with the results given in Section 5 we consider two different observation operators, one in which the full state is observed and one where the state at every other grid point is observed. We further assume that the true observation and background error statistics are homogeneous and are defined, as in Waller et al. (2016b), using a second order auto regressive function with length scales 5 and 10 respectively. For our first experiment we assume that we observe half the state and only know the observation error variance and hence neglect the correlations i.e. $\widetilde{\mathbf{R}}=\mathbf{I}$. For the second experiment we observe half the state, but this time use the correct $\mathbf{R}$ matrix. Finally we increase the observation density and observe all grid points and assume the correct $\mathbf{R}$ matrix.

In all three experiments the matrices $\mathbf{R}, \widetilde{\mathbf{R}}$ and $\mathbf{H B H}^{T}$ are circulant matrices. Since the sums, products and inverses of circulant matrices are circulant, $\mathbf{H A H} \mathbf{H}^{T}$ is also circulant. The eigenvalues of circulant matrices are positive and can be found using a discrete Fourier transform and consequently may be 867 ordered according to wave number. In this case the order of 868 the eigenvalues has a relation to the scales in the analysis error 869 in observation space. Therefore, the eigenvalues of the analysis 870 error covariance in observation space allows us to understand 871 the uncertainty we have at different scales in the analysis in 872 observation space. The $k^{t h}$ eigenvalue, $\phi_{k}$, of a circulant matrix 873 $\mathbf{C} \in \mathbb{R}^{N \times N}$ associated with frequency $\omega_{k}=\frac{2 \pi k}{\Delta_{x} N}$, and sampling 874 interval $\Delta_{x}$, is,

$$
\phi_{k}=\sum_{n=0}^{N-1} c_{n} e^{-\frac{2 \pi k n i}{N}}
$$

where $c_{n}$ is the $n^{\text {th }}$ coefficient of the first row of the circulant 876 matrix. In our experiments, due to the different number of 877 observations, the size of $\mathbf{H A H}^{T}$ changes. However, by analysing 878 the results as a function of wavenumber we can compare 879 physically consistent quantities. The results for our experiments 880 are plotted in Figure 8 and discussed in Section 5.

\section{Acknowledgement}

Susan P. Ballard passed away after a long illness on 12 July 2018, 883 during the manuscript drafting process.

J. A. Waller, S. L. Dance and N. K. Nichols were funded in part by 885 the NERC grants NE/K008900/1 Forecasting Rainfall exploiting 886 new data Assimilation techniques and Novel observations of 887 Convection (FRANC) and Towards END-to End flood forecasting 888 and a tool for ReaL-time catchment susceptibilitY (TENDERLY) 889 and in part by the NERC National Centre for Earth Observation. 890 The authors would also like to thank Alison Fowler, Ross 891 Bannister, Andrew Lorenc and Stephen Oxley for their valuable 892 discussions. 893 The data used in this study may be obtained on request, subject to 894 licensing conditions, by contacting the corresponding author.

Ballard SP, Li Z, Simonin D, Caron JF. 2016. Performance of 897 4D-Var NWP-based nowcasting of precipitation at the Met 898 Office for summer 2012. Quarterly Journal of the Royal 899 Meteorological Society 142(694): 472-487, doi:10.1002/qj. 900 2665. 
Bormann N, Bauer P. 2010. Estimates of spatial and interchannel observation-error characteristics for current sounder radiances for numerical weather prediction. I: Methods and application to ATOVS data. Quarterly Journal of the Royal Meteorological Society 136(649): 1036-1050, doi:10.1002/qj.616.

Bormann N, Bonavita M, Dragani R, Eresmaa R, Matricardi M, McNally A. 2016. Enhancing the impact of IASI observations through an updated observation-error covariance matrix. Quarterly Journal of the Royal Meteorological Society 142(697): 1767-1780, doi:10.1002/qj.2774.

Bormann N, Collard A, Bauer P. 2010. Estimates of spatial and interchannel observation-error characteristics for current sounder radiances for numerical weather prediction. II: Application to AIRS and IASI data. Quarterly Journal of the Royal Meteorological Society 136(649): 1051-1063, doi: 10.1002/qj.615.

Buehner M. 2010. Error statistics in data assimilation: Estimation and modelling. In: Data Assimilation Making Sense of Observations, Lahoz W, Khattatov B, Mènard R (eds), ch. 4, Springer, p. 99.

Campbell WF, Satterfield EA, Ruston B, Baker NL. 2017. Accounting for correlated observation error in a dualformulation $4 \mathrm{~d}$ variational data assimilation system. Monthly Weather Review 145(3): 1019-1032, doi: 10.1175/MWR-D-16-0240.1.

Caya A, Sun J, Snyder C. 2005. A comparison between the 4dvar and the ensemble kalman filter techniques for radar data assimilation. Monthly Weather Review 133(11): 3081-3094, doi:10.1175/MWR3021.1.

Clark P, Roberts N, Lean H, Ballard S, Charlton-Perez C. 2015. Convection-permitting models: A step-change in rainfall forecasting. Meteorological Applications 23(2): 165-181, doi: $10.1002 /$ met. 1538

Cordoba M, Dance S, Kelly G, Nichols N, Waller J. 2017. Diagnosing atmospheric motion vector observation errors for an operational high resolution data assimilation system. Quarterly Journal of the Royal Meteorological Society 143(702): 333341, doi:10.1002/qj.2925.
Courtier P, Thepaut J, Hollingsworth A. 1994. A strategy for 940 operational implementation of 4D-Var,using an incremental 941 approach. Quarterly Journal of the Royal Meteorological 942 Society 120(519): 1367-1387, doi:10.1002/qj.49712051912. 943

Daley R. 1991. Atmospheric data analysis. Cambridge University 944 Press: Cambridge, UK.

Davies T, Cullen MJP, Malcolm AJ, Mawson MH, Staniforth 946 A, White AA, Wood N. 2005. A new dynamical core 947 for the met office's global and regional modelling of the 948 atmosphere. Quarterly Journal of the Royal Meteorological 949 Society 131(608): 1759-1782, doi:10.1256/qj.04.101.

Davis C, Brown B, Bullock R. 2006. Object-based verification of 951 precipitation forecasts. part i: Methodology and application to 952 mesoscale rain areas. Monthly Weather Review 134(7): 1772- 953 1784, doi:10.1175/MWR3145.1.

de Haan S, Stoffelen A. 2012. Assimilation of high-resolution 955 mode-s wind and temperature observations in a regional nwp 956 model for nowcasting applications. Weather and Forecasting 957 27(4): 918-937, doi:10.1175/WAF-D-11-00088.1.

Descombes G, Auligné T, Vandenberghe F, Barker DM. 959 2015. Generalized background error covariance matrix model 960 (GEN_BE v2.0). Geosci. Model Dev. 8(3): 669-696, doi:10. 961 5194/gmd-8-669-2015.

Desroziers G, Berre L, Chapnik B, Poli P. 2005. Diagnosis 963 of observation, background and analysis-error statistics 964 in observation space. Quarterly Journal of the Royal 965 Meteorological Society 131(613): 3385-3396, doi:10.1256/qj. 966 05.108 .

Dixon M, Li Z, Lean H, Roberts N, Ballard S. 2009. Impact 968 of data assimilation on forecasting convection over the united 969 kingdom using a high-resolution version of the met office 970 unified model. Monthly Weather Review 137(5): 1562-1584, 971 doi:10.1175/2008MWR2561.1.

Droegmeier K. 1997. The numerical prediction of thunderstorms: 973 Challenges, potential benefit, and results from real-time 974 operational test. WMO Bulletin 46(4): 324-336.

Ebert EE, Damrath U, Wergen W, Baldwin ME. 2003. The WGNE 976 assessment of short-term quantitative precipitation forecasts. 977 Bulletin of the American Meteorological Society 84: 481492. 
Ebert EE, MacBride JL. 2000. Verification of precipitation in weather systems: Determination of the systematic errors. $J$. Hydrol. 239: 179202.

Fisher M, Andersson E. 2001. Developments in 4D-Var and Kalman Filtering. Technical report, ECMWF. ECMWF Technical Memorandum 347.

Fowler AM, Dance SL, Waller JA. 2018. On the interaction of observation and prior error correlations in data assimilation. Quarterly Journal of the Royal Meteorological Society 144(710): 48-62, doi:10.1002/qj.3183.

Gao J, Stensrud DJ. 2012. Assimilation of reflectivity data in a convective-scale, cycled 3dvar framework with hydrometeor classification. Journal of the Atmospheric Sciences 69(3): 1054-1065, doi:10.1175/JAS-D-11-0162.1.

Gilleland EB, Ahijevych D, Brown BG, B C, E EE. 2009. Intercomparison of spatial forecast verification methods. Weather Forecast 24: 1416-1430, doi:10.1175/2009WAF2222269.1.

Golub GH, Van Loan CF. 1996. Matrix computations. Baltimore: The Johns Hopkins University Press, third edn.

Healy SB, White AA. 2005. Use of discrete Fourier transforms in the 1D-Var retrieval problem. Quarterly Journal of the Royal Meteorological Society 131(605): 63-72, doi:10.1256/qj. 03.193 .

Ide k, Courtier P, Ghil M, Lorenc AC. 1997. Unified notation for data assimilation: Operational, sequential and variational. Journal of the Meteorological Society of Japan 75(1B): 181189, doi:n/a.

Janjić T, Bormann N, Bocquet M, Carton JA, Cohn SE, Dance SL, Losa SN, Nichols NK, Potthast R, Waller JA, Weston P. 2017. On the representation error in data assimilation. Quarterly Journal of the Royal Meteorological Society n/a(n/a): n/a - n/a, doi:10.1002/qj.3130.

Johnson A, Wang X. 2013. Object-based evaluation of a storm scale ensemble during the 2009 noaa hazardous weather testbed spring experiment. Mon. Wea. Rev. 141(n/a): 1079-1098.

Jones CD, Macpherson B. 1997. A latent heat nudging scheme for the assimilation of precipitation data into an operational mesoscale model. Meteorological Applications 4(3): 269277.
Lange H, Janjić T. 2016. Assimilation of Mode-S EHS aircraft 1017 observations in COSMO-KENDA. Monthly Weather Review 1018 144(5): 1697-1711, doi:10.1175/MWR-D-15-0112.1, URL 1019 http://dx.doi.org/10.1175/MWR-D-15-0112.1. 1020

Lean H, Clark P, Dixon M, Roberts N, Fitch A, Forbes R, 1021 Halliwell C. 2008. Charictaristics of high-resolution versions of 1022 the Met Office Unified Model for forecasting convection over 1023 the United Kingdom. Monthly Weather Review 136(9): 3408- 1024 3424, doi:10.1175/2008MWR2332.1.

Liu ZQ, Rabier F. 2003. The potential of high-density 1026 observations for numerical weather prediction: A study 1027 with simulated observations. Quarterly Journal of the Royal 1028 Meteorological Society 129(594): 3013-3035, doi:10.1256/qj. 1029 02.170 .

Lorenc AC, Ballard SP, Bell RS, Ingleby NB, Andrews PLF, 1031 Barker DM, Bray JR, Clayton AM, Dalby T, Li D, Payne TJ, 1032 Saunders FW. 2000. The met. office global three-dimensional 1033 variational data assimilation scheme. Quarterly Journal of the 1034 Royal Meteorological Society 126(570): 2991-3012, doi:10. 1035 1002/qj.49712657002.

1036

Lorenc AC, Jardak M. 2018. A comparison of hybrid variational 1037 data assimilation methods for global nwp. Quarterly Journal of 1038 the Royal Meteorological Society 0: In press, doi:10.1002/qj. 1039 3401.

Lorenc AC, Rawlins F. 2005. Why does 4D-Var beat 3D- 1041 Var? Quarterly Journal of the Royal Meteorological Society 1042 131(613): 3247-3257, doi:10.1256/qj.05.85.

Mittermaier MP, Roberts N. 2010. Inter-comparison of spatial 1044 forecast verification methods: Identifying skillful spatial scales 1045 using the fractions skill score. Wea. Forecasting 25: 343-354. 1046 Pacheco PS. 1997. Parallel programming with mpi. Morgan 1047 Kaufmann: San Francisco, USA.

Piccolo C, Cullen M. 2011. Adaptive mesh method in the Met 1049 Office variational data assimilation system. Quarterly Journal 1050 of the Royal Meteorological Society 137(656): 631-640, doi: 1051 10.1002/qj.801.

Piccolo C, Cullen M. 2012. A new implementation of the adaptive 1053 mesh transform in the Met Office 3D-Var system. Quarterly 1054 
Journal of the Royal Meteorological Society 138(667): 15601570, doi:10.1002/qj.1880.

Rainwater S, Bishop CH, Campbell WF. 2015. The benefits of correlated observation errors for small scales. Quarterly Journal of the Royal Meteorological Society 141(693): 34393445, doi:10.1002/qj.2582.

Rawlins F, Ballard SP, Bovis KJ, Clayton AM, Li D, Inverarity GW, Lorenc AC, Payne TJ. 2007. The Met Office global fourdimensional variational data assimilation scheme. Quarterly Journal of the Royal Meteorological Society 133(623): 347362, doi:10.1002/qj.32.

Roberts N. 2008. Assessing the spatial and temporal variation in the skill of precipitation forecasts from an nwp model. Meteorological Applications, Special Issue: Forecast Verification 15(1): 1631697.

Roberts N, Lean H. 2008. Scale-selective verification of rainfall accumulations from high-resolution forecasts of convective events. Monthly Weather Review 136(1): 78-97, doi:10.1175/ 2007MWR2123.1.

Rodwell MJ, Palmer TN. 2007. Using numerical weather prediction to assess climate models. Quarterly Journal of the Royal Meteorological Society 133(622): 129-146, doi:10.1002/ qj.23.

Salonen K, Jrvinen H, Eresmaa R, Niemel S. 2007. Bias estimation of doppler-radar radial-wind observations. Quarterly Journal of the Royal Meteorological Society 133(627): 15011507, doi:10.1002/qj.114.

Seaman R. 1977. Absolute and differential accuracy of analyses achievable with specified observation network characteristics. Monthly Weather Review 105(10): 12111222, doi:10.1175/ 1520-0493(1977)105〈1211:AADAOA $\rangle 2.0 . C O ; 2$.

Simonin D, Ballard SP, Li Z. 2014. Doppler radar radial wind assimilation using an hourly cycling 3d-var with a 1.5 $\mathrm{km}$ resolution version of the met office unified model for nowcasting. Quarterly Journal of the Royal Meteorological Society 140(684): 2298-2314, doi:10.1002/qj.2298.

Skok G. 2015. Analysis of fraction skill score properties for a displaced rainband in a rectangular domain. Met. Apps 22: 477484, doi:10.1002/qj.2298.
Stewart LM. 2010. Correlated observation errors 1094 in data assimilation. PhD thesis, University of 1095 Reading. Http://www.reading.ac.uk/maths-and- 1096 stats/research/theses/maths-phdtheses.aspx.

Stewart LM, Cameron J, Dance SL, English S, 1098 Eyre JR, Nichols NK. 2009. Observation error 1099 correlations in IASI radiance data. Technical report, 1100 University of Reading. Mathematics reports series, 1101 www.reading.ac.uk/web/FILES/maths/obs_error_IASI_radiance.pdd62

Stewart LM, Dance SL, Nichols NK. 2008. Correlated observation 1103 errors in data assimilation. International Journal for Numerical 1104 Methods in Fluids 56(8): 1521-1527, doi:10.1002/fld.1636. 1105

Stewart LM, Dance SL, Nichols NK. 2013. Data assimilation with 1106 correlated observation errors: experiments with a 1-D shallow 1107 water model. Tellus A 65(1): 19546, doi:10.3402/tellusa.v65i0. 1108 19546.

Stewart LM, Dance SL, Nichols NK, Eyre JR, Cameron J. 2014. 1110 Estimating interchannel observation-error correlations for IASI 1111 radiance data in the Met Office system. Quarterly Journal of 1112 the Royal Meteorological Society 140(681): 1236-1244, doi: 1113 10.1002/qj.2211.

Strajnar B, agar N, Berre L. 2015. Impact of new aircraft 1115 observations mode-s mrar in a mesoscale nwp model. Journal 1116 of Geophysical Research: Atmospheres 120(9): 3920-3938, 1117 doi:10.1002/2014JD022654.

Sun J, Xue M, Wilson JW, Zawadzki I, Ballard SP, Onvlee- 1119 Hooimeyer J, Joe P, Barker DM, Li PW, Golding B, Xu 1120 M, Pinto J. 2014. Use of NWP for nowcasting convective 1121 precipitation: Recent progresses and challenges. Bulletin of the 1122 American Meteorological Society 95(3): 409-426, doi:10.1175/ 1123 BAMS-D-11-00263.1.

Tabeart J, Dance SL, Haben SA, Lawless AS, Nichols NK, 1125 Waller JA. 2018. The conditioning of least squares problems 1126 in variational data assimilation. Numerical Linear Algebra with 1127 Applications doi:10.1002/nla.2165.

Tang Y, Lean HW, Bornemann J. 2013. The benefits of the 1129 Met Office variable resolution NWP model for forecasting 1130 convection. Meteorological Applications 20(4): 417-426, doi: 1131 10.1002/met.1300. 
Velden C, Lewis WE, Bresky W, Stettner D, Daniels J, Wanzong S. 2017. Assimilation of high-resolution satellitederived atmospheric motion vectors: Impact on hwrf forecasts of tropical cyclone track and intensity. Monthly Weather Review 145(3): 1107-1125, doi:10.1175/MWR-D-16-0229.1.

Waller JA, Ballard SP, Dance SL, Kelly G, Nichols NK, Simonin D. 2016a. Diagnosing horizontal and inter-channel observation error correlations for SEVIRI observations using observationminus-background and observation-minus-analysis statistics. Remote Sensing 8(7): 581, doi:10.3390/rs8070581.

Waller JA, Dance SL, Lawless AS, Nichols NK, Eyre JR. 2014. Representativity error for temperature and humidity using the Met Office high-resolution model. Quarterly Journal of the Royal Meteorological Society 140(681): 1189-1197, doi:10. 1002/qj.2207.

Waller JA, Dance SL, Nichols NK. 2016b. Theoretical insight into diagnosing observation error correlations using observationminus-background and observation-minus-analysis statistics. Quarterly Journal of the Royal Meteorological Society 142: 418-431, doi:10.1002/qj.2661.

Waller JA, Simonin D, Dance SL, Nichols NK, Ballard SP. 2016c. Diagnosing observation error correlations for Doppler radar radial winds in the Met Office UKV model using observationminus-background and observation-minus-analysis statistics. Monthly Weather Review 144(10): 3533-3551, doi:10.1175/ MWR-D-15-0340.1.

Wang Y, Wang X. 2017. Direct assimilation of radar reflectivity without tangent linear and adjoint of the nonlinear observation operator in the gsi-based envar system: Methodology and experiment with the 8 may 2003 oklahoma city tornadic supercell. Monthly Weather Review 145(4): 1447-1471, doi: 10.1175/MWR-D-16-0231.1.

Wattrelot E, Caumont O, Mahfouf JF. 2014. Operational implementation of the $1 \mathrm{~d}+3 \mathrm{~d}$-var assimilation method of radar reflectivity data in the arome model. Monthly Weather Review 142(5): 1852-1873, doi:10.1175/MWR-D-13-00230.1.

Weston P. 2011. Progress towards the implementation of correlated observation errors in 4D-Var. Technical report, Met Office, UK. Forecasting Research Technical Report 560. in the assimilation of high-resolution sounder data. Quarterly 1173 Journal of the Royal Meteorological Society 140(685): 2420- 1174 2429, doi:10.1002/qj.2306.

Wlasak MA, Cullen MJF. 2014. Modelling static 3d spatial 1176 background error covariances - the effect of vertical and 1177 horizontal transform order. Advances in Science and Research 1178 11(1): 63-67, doi:10.5194/asr-11-63-2014. 1179

Yaremchuk M, D’Addezio JM, Panteleev G, Jacobs G. 2018. On 1180 the approximation of the inverse error covariances of high- 1181 resolution satellite altimetry data. Quarterly Journal of the 1182 Royal Meteorological Society: In pressdoi:10.1002/qj.3336. $\quad 1183$ $\mathrm{g}$ 
Table 1. Experiment details

\begin{tabular}{|c|c|c|}
\hline Experiment & $\begin{array}{l}\text { Doppler wind observation } \\
\text { error matrix }\end{array}$ & $\begin{array}{l}\text { Doppler wind super-observation } \\
\text { thinning distance }\end{array}$ \\
\hline Control & $\begin{array}{l}\text { Diagonal observation error } \\
\text { matrix (Operational) }\end{array}$ & $6 \mathrm{~km}$ \\
\hline Corr-R-6km & $\begin{array}{l}\text { Correlation observation } \\
\text { error matrix }\end{array}$ & $6 \mathrm{~km}$ \\
\hline Corr-R-3km & $\begin{array}{l}\text { Correlation observation } \\
\text { error matrix }\end{array}$ & $3 \mathrm{~km}$ \\
\hline Diag-R-3km & $\begin{array}{l}\text { Diagonal observation error } \\
\text { matrix (Operational) }\end{array}$ & $3 \mathrm{~km}$ \\
\hline
\end{tabular}

Table 2. Trial average $(\mu)$ and standard deviation $(\sigma)$ of various parameters measuring the performance of the assimilation system.

\begin{tabular}{lcccccccc}
\hline \multirow{2}{*}{ Experiments } & \multicolumn{2}{c}{ Iteration count } & \multicolumn{2}{c}{ Evaluation count } & \multicolumn{2}{c}{$J_{b}$} & \multicolumn{2}{c}{$J_{O}$} \\
\cline { 2 - 9 } & $\mu$ & $\sigma$ & $\mu$ & $\sigma$ & $\mu$ & $\sigma$ & $\mu$ & $\sigma$ \\
\hline \hline Control & 27.4 & 15.1 & 40.8 & 23.5 & 1752.16 & 526.1 & 9207.53 & 2707.16 \\
Corr-R-6km & 27.7 & 14.6 & 41.6 & 22.8 & 1722.43 & 500.37 & 9235.66 & 2732.84 \\
Corr-R-3km & 28.2 & 14.4 & 40.9 & 21.6 & 2050.53 & 761.25 & 10134.63 & 3175.32 \\
Diag-R-3km & 29.1 & 14.9 & 41.5 & 23.8 & 2277.59 & 910.74 & 9679.28 & 2900.41 \\
\hline
\end{tabular}

Table 3. Computational cost in seconds. The first row shows the trial average wall-clock time $\left(\bar{W}^{(\text {trial })}\right)$. Subsequent rows show the average wall-clock time $\left(\bar{W}^{(10)}\right)$, the average cost for calculating $J\left(\bar{J}^{(10)}\right)$, and the average cost for calculating $J_{o}\left(\bar{J}_{o}^{(10)}\right)$, over 10 iterations for the $12 \mathrm{Z}$ run on the 7 th of April 2016.

\begin{tabular}{lccc}
\hline & Control & Corr-R-6km & Corr-R-3km \\
\hline \hline $\bar{W}^{(\text {trial })}[\mathrm{s}]$ & 272 & 293 & 288 \\
\hline $\bar{W}^{(10)}[\mathrm{s}]$ & 73 & 72 & 73 \\
$\bar{J}^{(10)}[\mathrm{s}]$ & 22.16 & 23.83 & 23.43 \\
$\bar{J}_{o}^{(10)}[\mathrm{s}]$ & 0.81 & 2.21 & 2.23 \\
\hline
\end{tabular}

Table 4. Surface verification scores and UK NWP index. All the values are presented as a percentage (positive values show improvement over the Control).

\begin{tabular}{lcc}
\hline Score & Corr-R-6km & Corr-R-3km \\
\hline \hline Visibility & +0.027 & +0.046 \\
Precipitation & -0.063 & -0.050 \\
Cloud cover & +0.047 & +0.012 \\
Cloud base height & -0.013 & -0.005 \\
$1.5 \mathrm{~m}$ temperature & -0.014 & +0.005 \\
10 m wind & +0.010 & +0.013 \\
\hline UK index & -0.005 & +0.021 \\
\hline
\end{tabular}




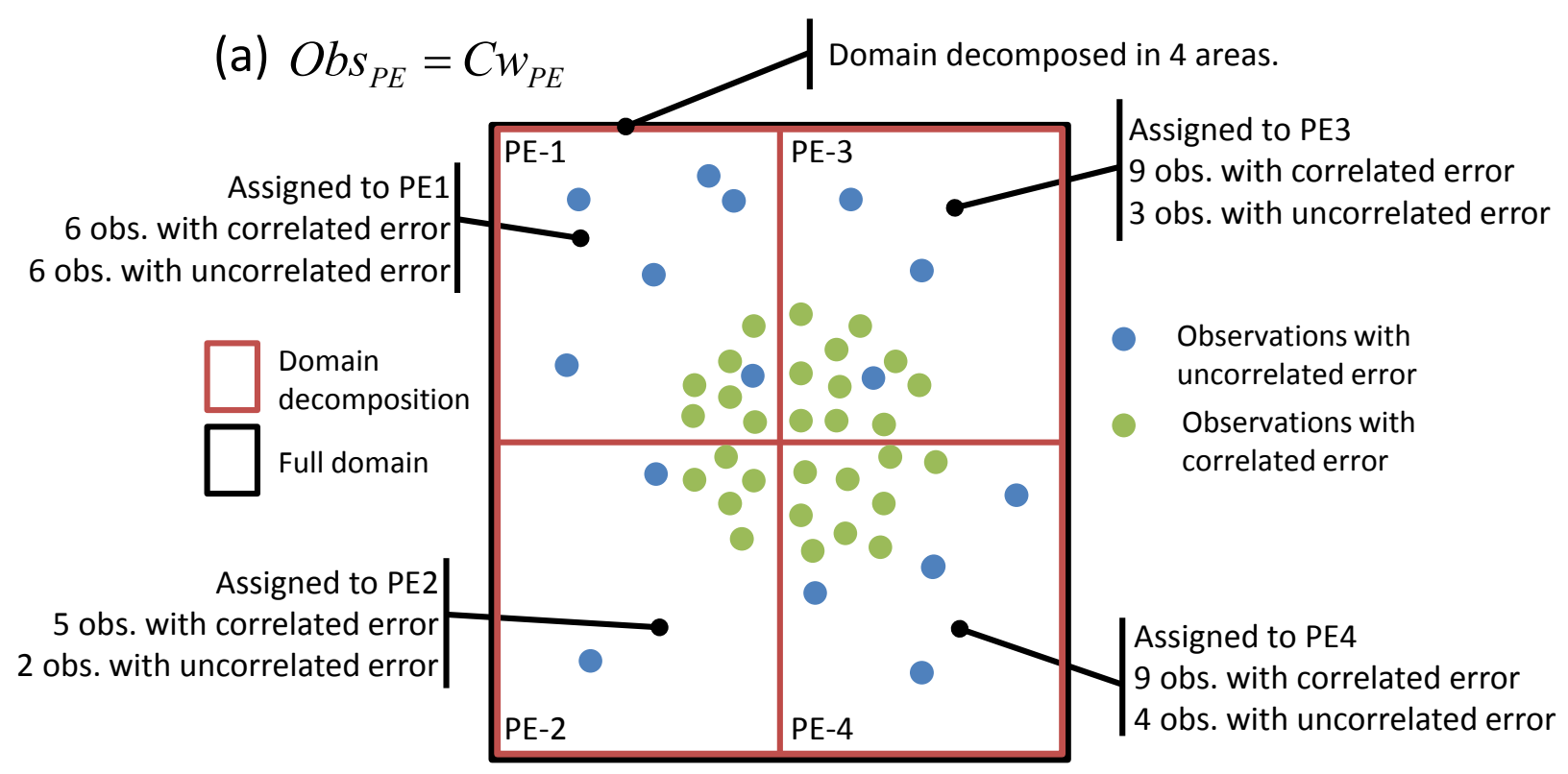

(b) $O b s_{P E} \neq C w_{P E}$

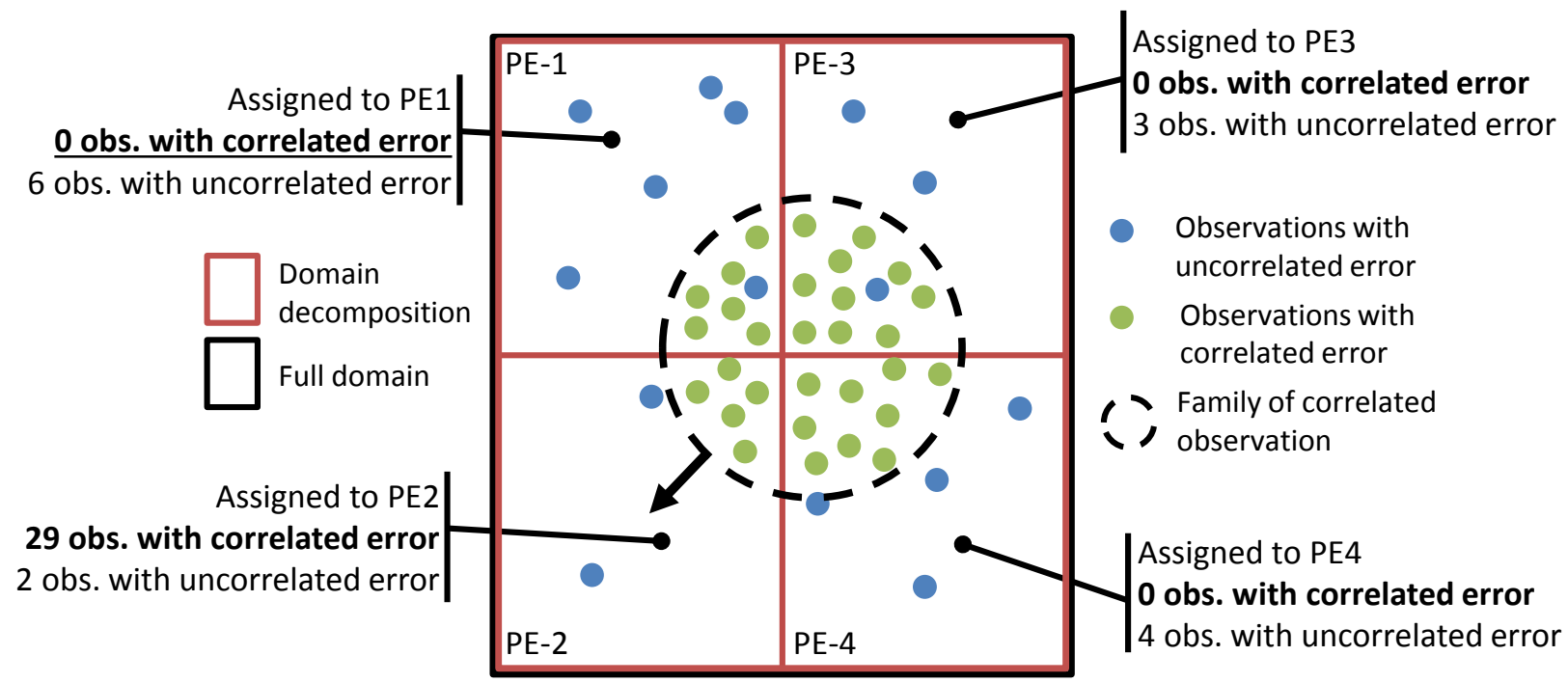

Figure 1. Example of the observation parallelisation in VAR for two observation types (uncorrelated and correlated error) with a 4 PE decomposition. (a) Conventional approach, i.e. without accounting for the horizontal correlation of the observation error. Each observation (with uncorrelated and correlated errors) is distributed between the $4 \mathrm{PE}$ according to its geographical location. (b) The new approach i.e. accounting for the horizontal correlation of the observation error. As before, each observation with uncorrelated error statistics is distributed between the $4 \mathrm{PE}$ according to its geographical location. However, this time all the observations with mutually correlated errors are assigned to a single family and sent to PE 2 regardless of their physical location. In both panels the model columns $\left(C_{w}\right)$ are distributed according to their geographical location. This implies that the distribution of each $C_{w}$ and observation is equivalent in (a) that is $O b s_{P E}=C w_{P E}$, and different in (b) i.e: $O b s_{P E} \neq C w_{P E}$. 


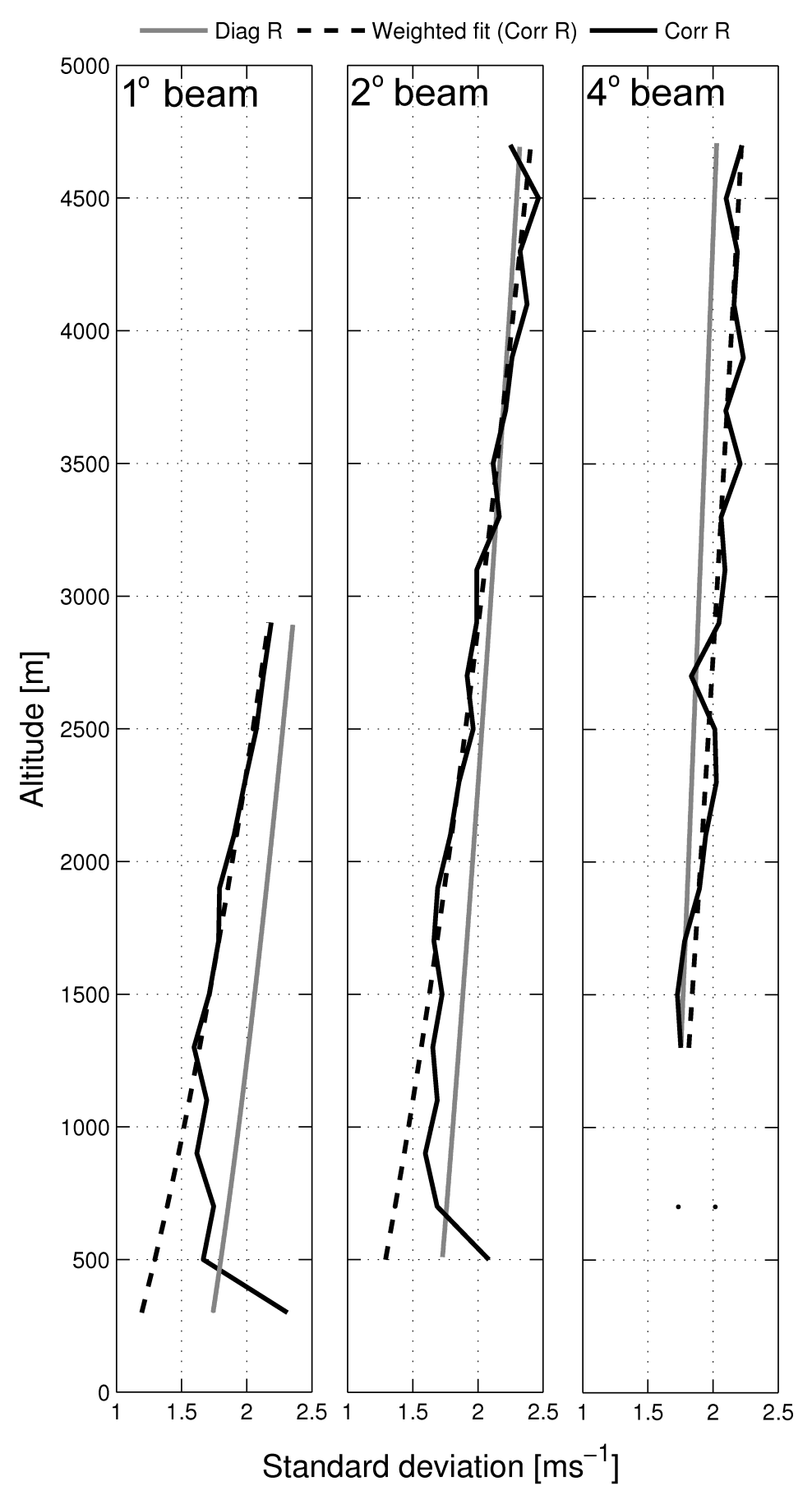

Figure 2. Error variance as function of height for three radar's beam elevation $\left(1^{\circ}, 2^{\circ}\right.$ and $\left.4^{\circ}\right)$. (Grey curve) operational error variance used in the Control experiment when the observation error covariance matrix is assumed to be diagonal. (Black curve) error variance for the diagnosed correlated observation error covariance matrix. (Black dash curve) weighted least square fit of the error variance for the diagnosed correlated observation error covariance matrix used in the Corr-R-6km and Corr-R-3km experiments. 

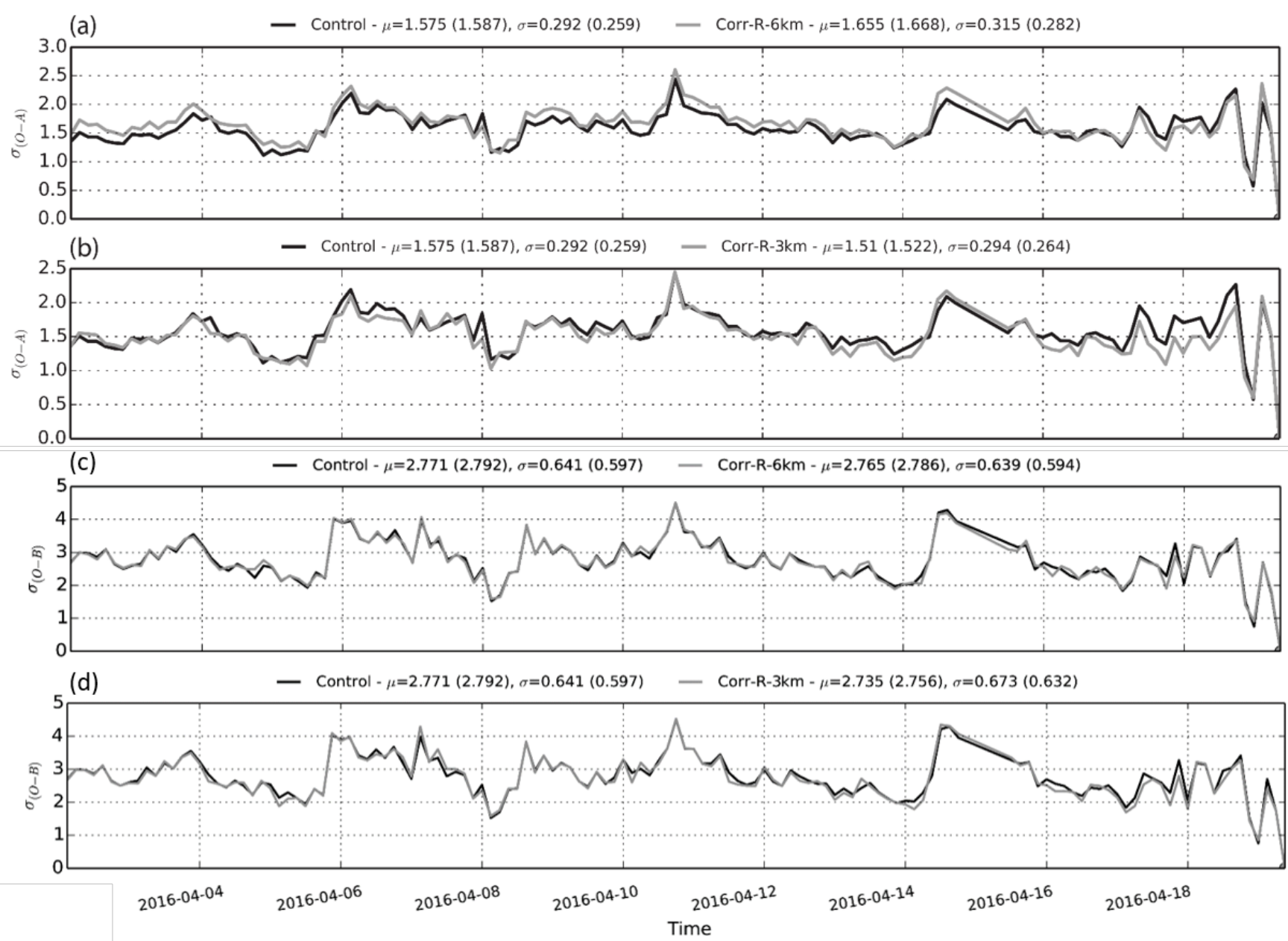

Figure 3. Time series standard deviation of Doppler radial wind $O-A(\mathrm{a}, \mathrm{b})$ and $O-B(\mathrm{c}, \mathrm{d})$ for the Control and Corr-R-6km (a,c) and for the Control and Corr-R-3km $(\mathrm{b}, \mathrm{d})$. In both panels the Control is in black and the experiment in grey. 
- Control - Corr-R-6km - Corr-R-3km

(a) Max value of the $x_{-}$wind increment

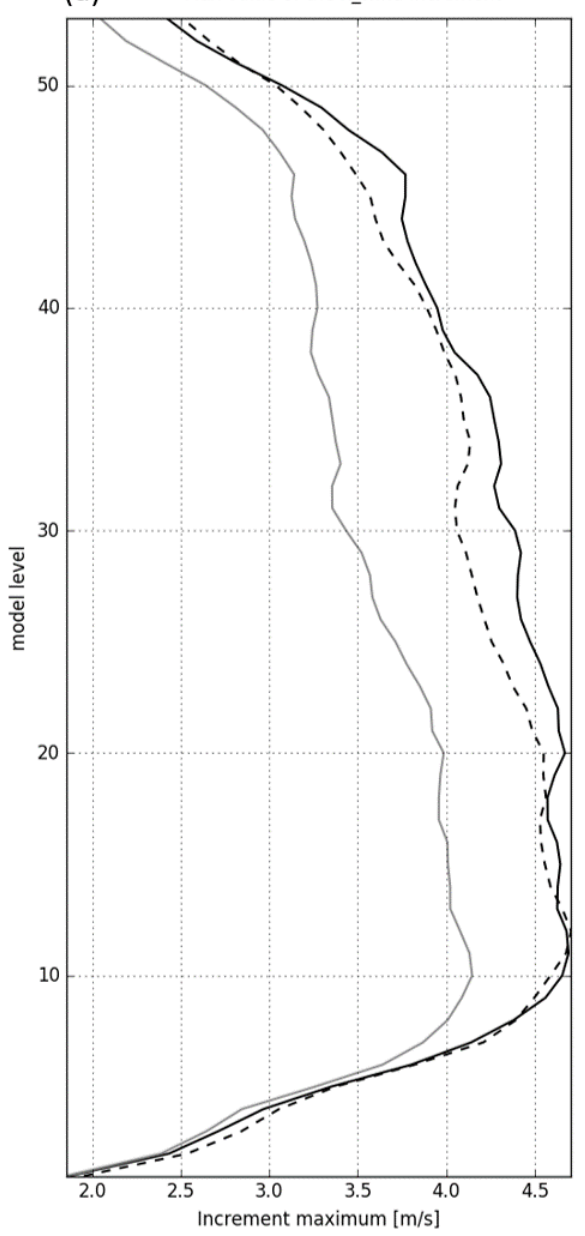

(b)

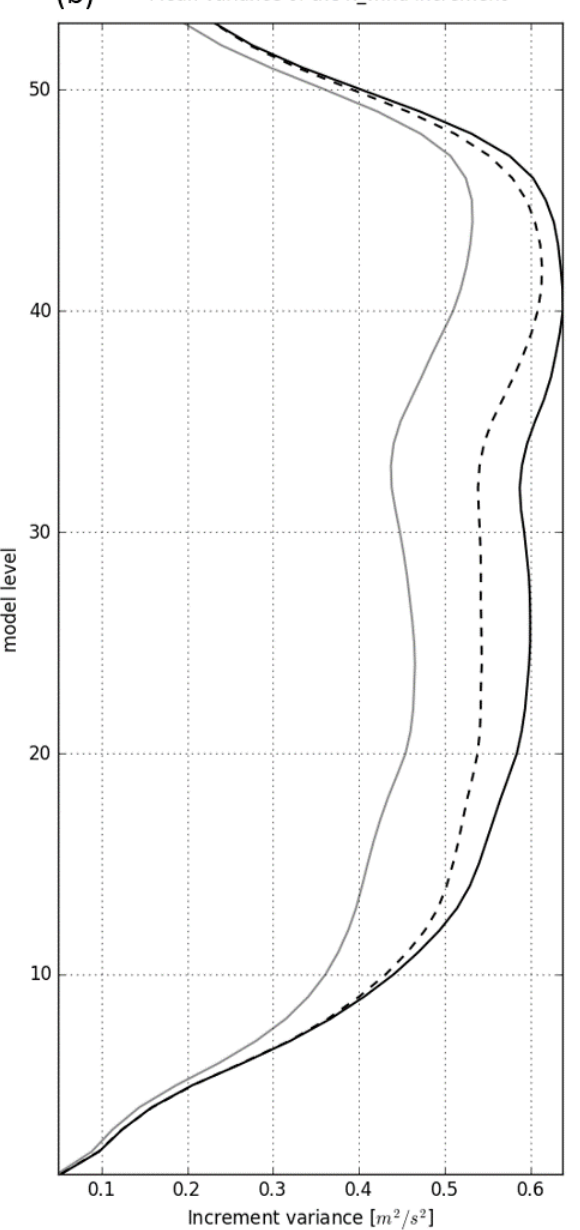

(c) Mean length scale of the $x_{-}$wind increment

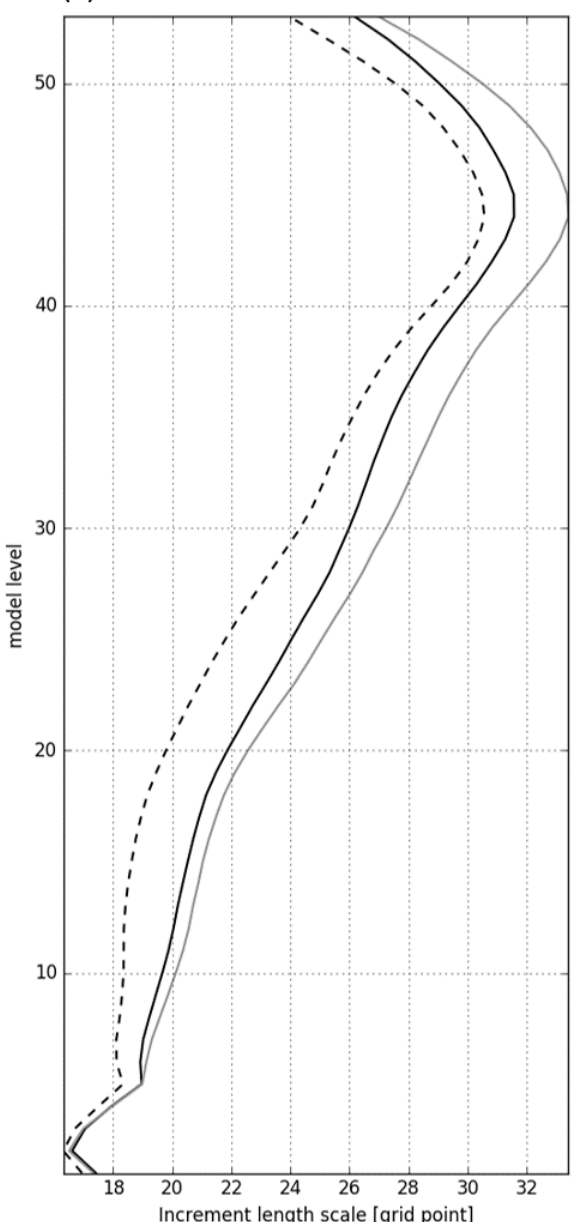

Figure 4. Trial average (a) maximum (b) variance and (c) length-scale (eq 6) for the zonal wind increment against model levels. (Black Curve) Control experiment; (grey curve) Corr-R-6km experiment and (black dash curve) Corr-R-3km experiment. 

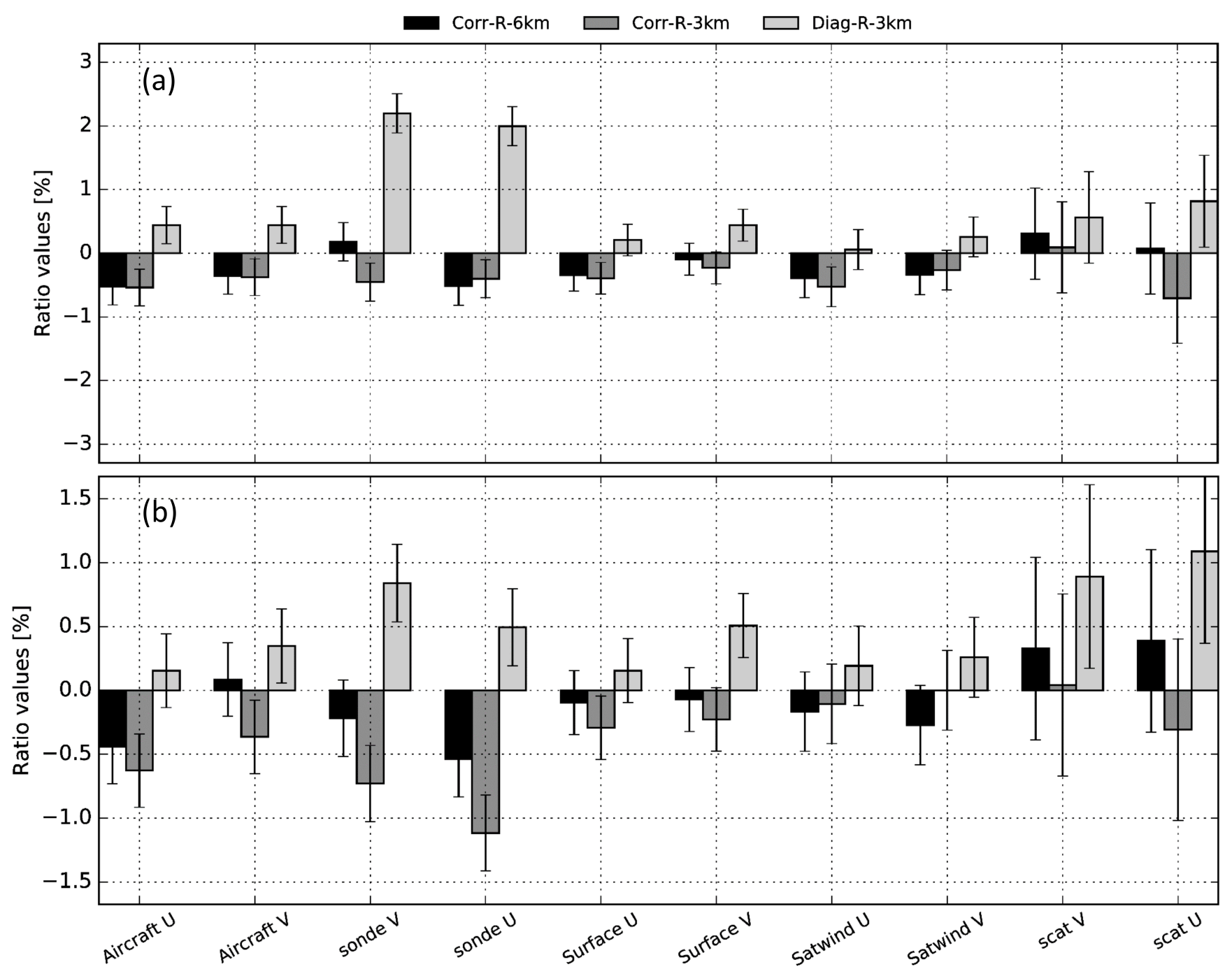

Figure 5. Wind observations (a) $O-A$ and (b) $O-B$ trial average standard deviation ratio between the experiments and the Control expressed as percentage and scaled to show positive impact as negative values (i.e. $\frac{\bar{\sigma}(O-A)_{e x p}}{\sigma_{(O-A)_{c t r l}}}-1$ and $\left.\frac{\bar{\sigma}(O-B)_{e x p}}{\sigma_{(O-B)_{c t r l}}}-1\right)$. In black exp $=$ Corr-R-6km, in dark grey exp $=$ Corr-R-3km and in light grey exp $=$ Diag-R-3km. The error bars represent the $95 \%$ confidence level. 


\section{Simonin et al.}
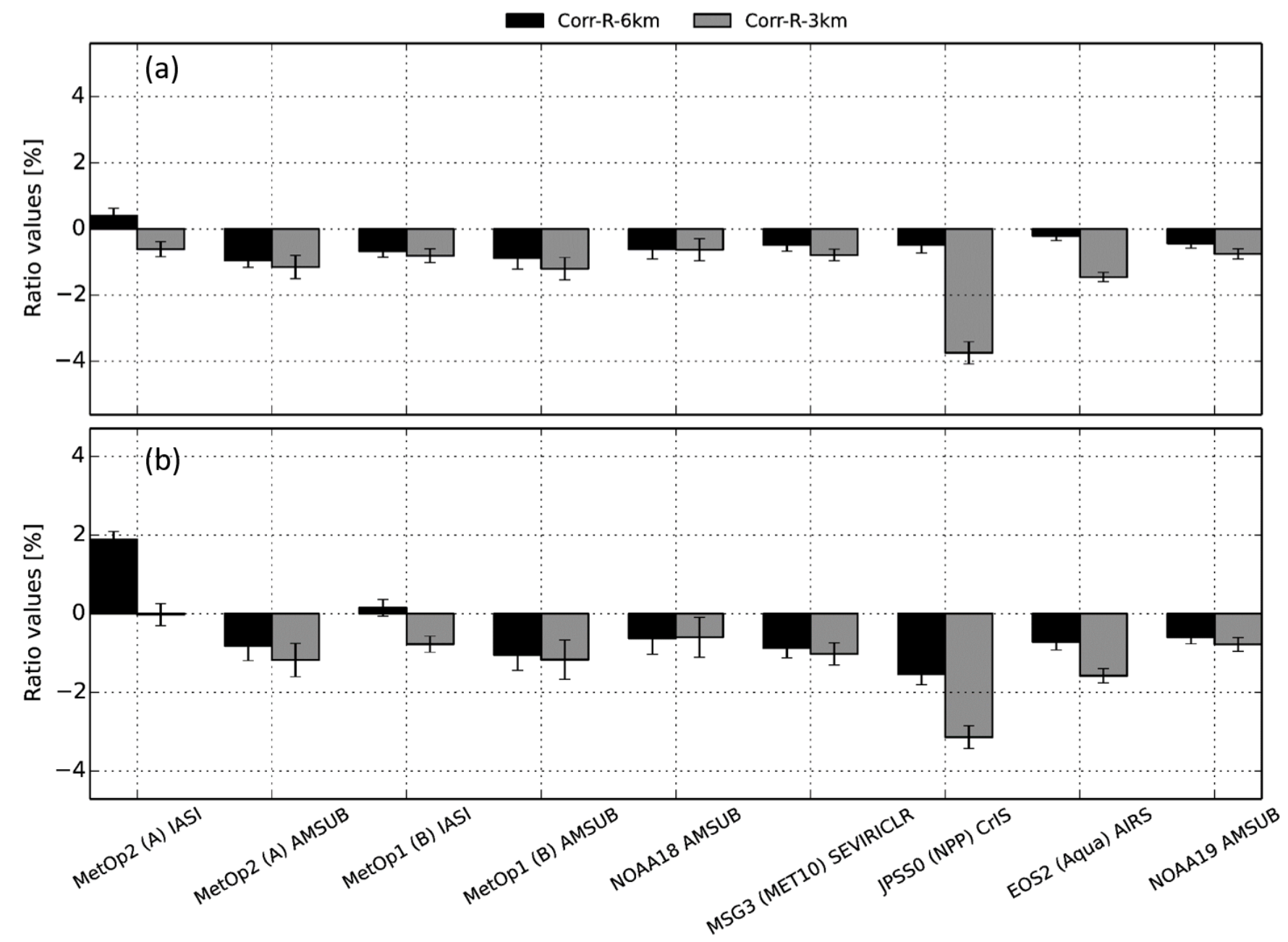

Figure 6. Similar to 5 but for Satellite observations. In black $e x p=$ Corr-R-6km and in dark grey exp $=$ Corr-R-3km. 

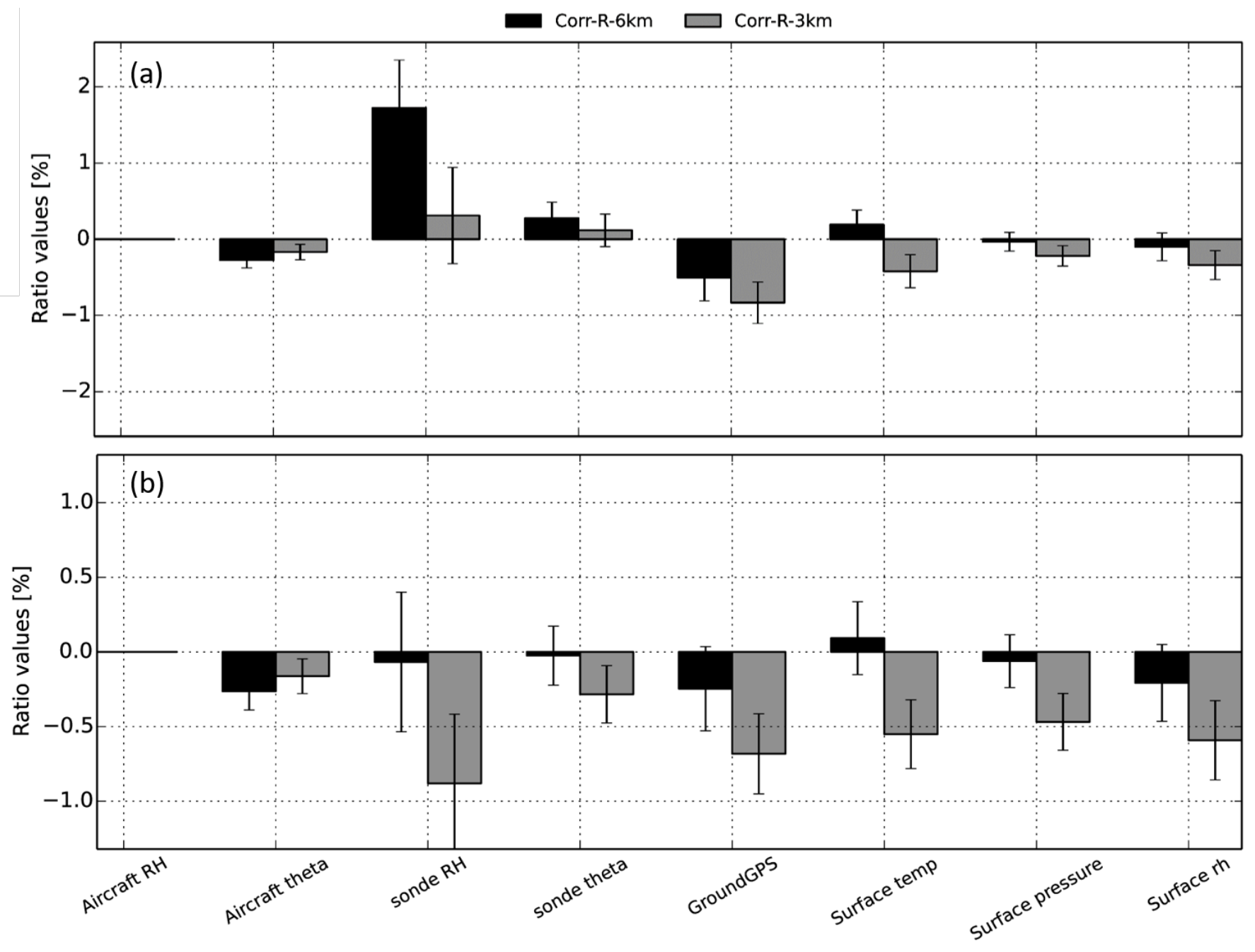

Figure 7. Similar to 6 but for the rest of the observations used. 


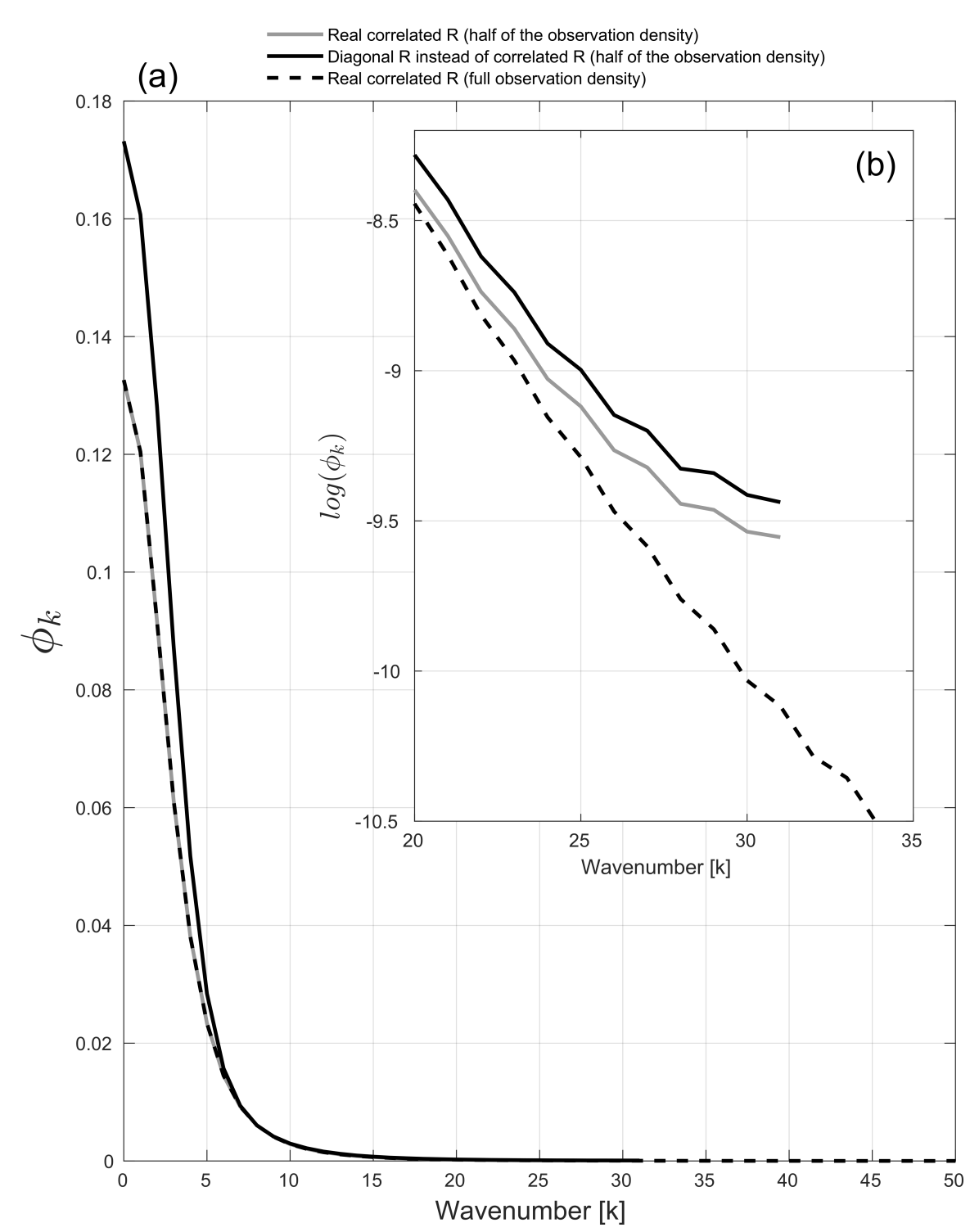

Figure 8. Panel (a) shows the eigenvalues of the analysis error covariance matrix $\left(\phi_{k}\right)$ in observation space (see appendix for details) against wavenumber. Insert (b) shows $\phi_{k}$ in log space for wavenumber ranging from 20 to 35. (Black curve) $\Phi_{k}$ using a diagonal observation error covariance when the true observation error covariance contains some correlation with the state being half observed. (Grey curve) $\phi_{k}$ using a correlated observation error covariance with the state being half observed. (Black dashed curve) $\phi_{k}$ using a correlated observation error covariance with the state being fully observed. 
$\Delta F S S=F S S_{E x p}-F S S_{C t r l}$

(a) $\max |\triangle F S S|=0.009$ [Ctrl: Control, Exp:Corr-R-6km]

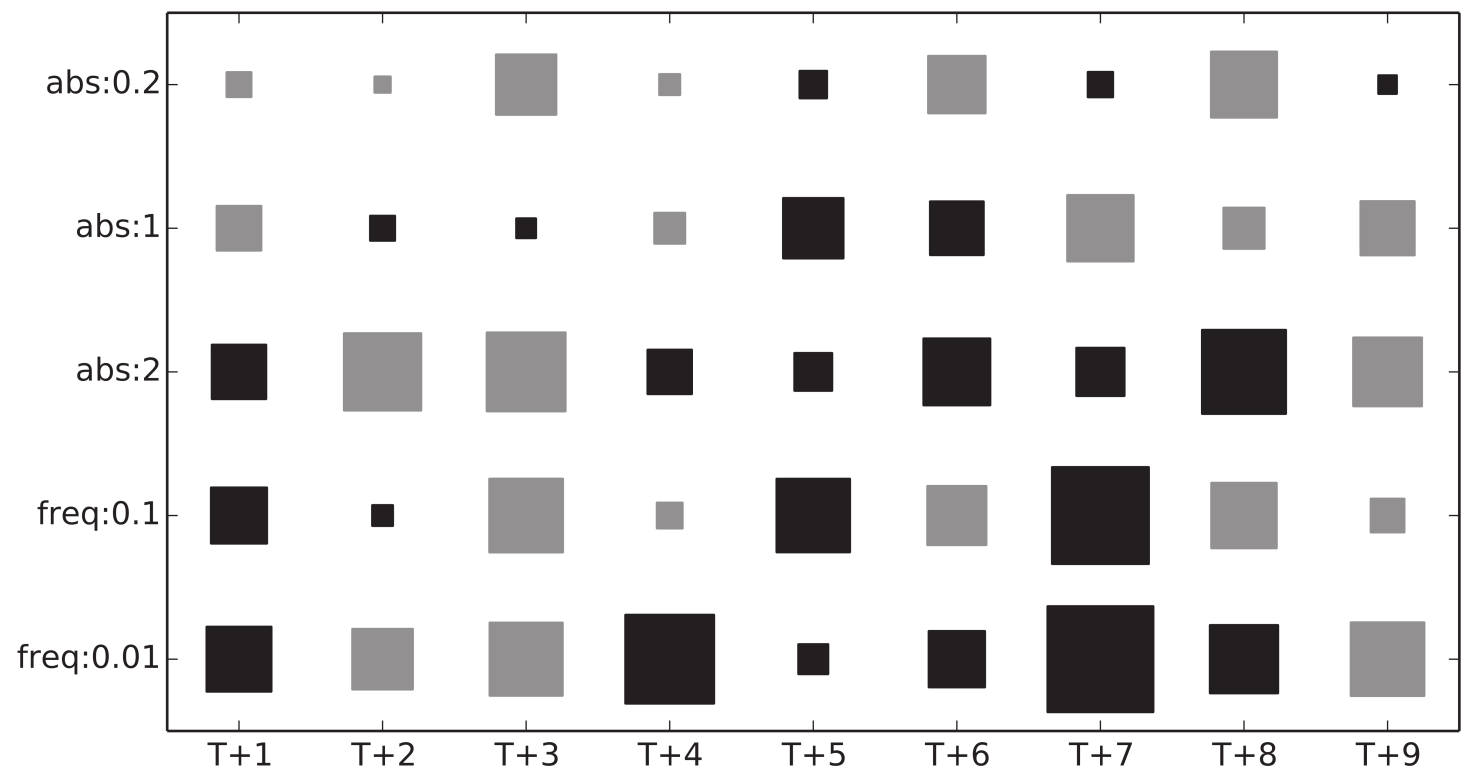

(b) $\max |\triangle F S S|=0.03$ [Ctrl: Control, Exp:Corr-R-3km]

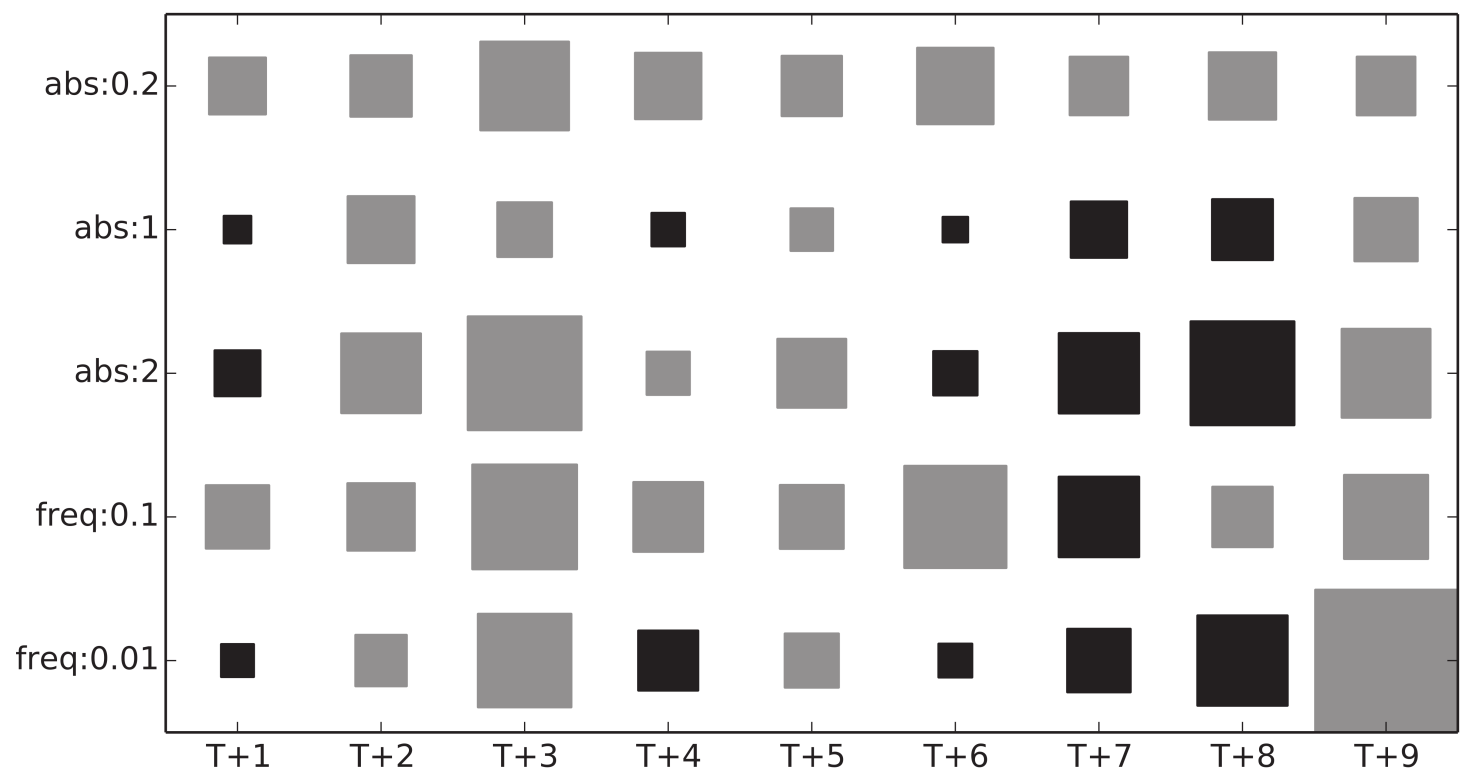

Figure 9. Hinton diagram showing the trial average FSS differences between the Corr-R-6km and the Control experiment (a) and between the Corr-R-3km and the Control experiment (b) for different forecast lead time and hourly rainfall accumulation thresholds with a neighborhood size of 41 grid-boxes. The sign and the amplitude of the change in FSS are shown with the color and size of the square respectively: positive values (positive impact) are shown as gray squares, whereas negative values (negative impact) are shown as blacks square. The rainfall accumulation thresholds on y-axis are $0.2 m m h^{-1}(a b s: 0.2), 1.0 m m h^{-1}(a b s: 1), 2.0 m m h^{-1}(a b s: 2)$, the $90^{t h}$ percentiles (freq:0.1) and the $99^{t h}$ percentiles (freq:0.01). 

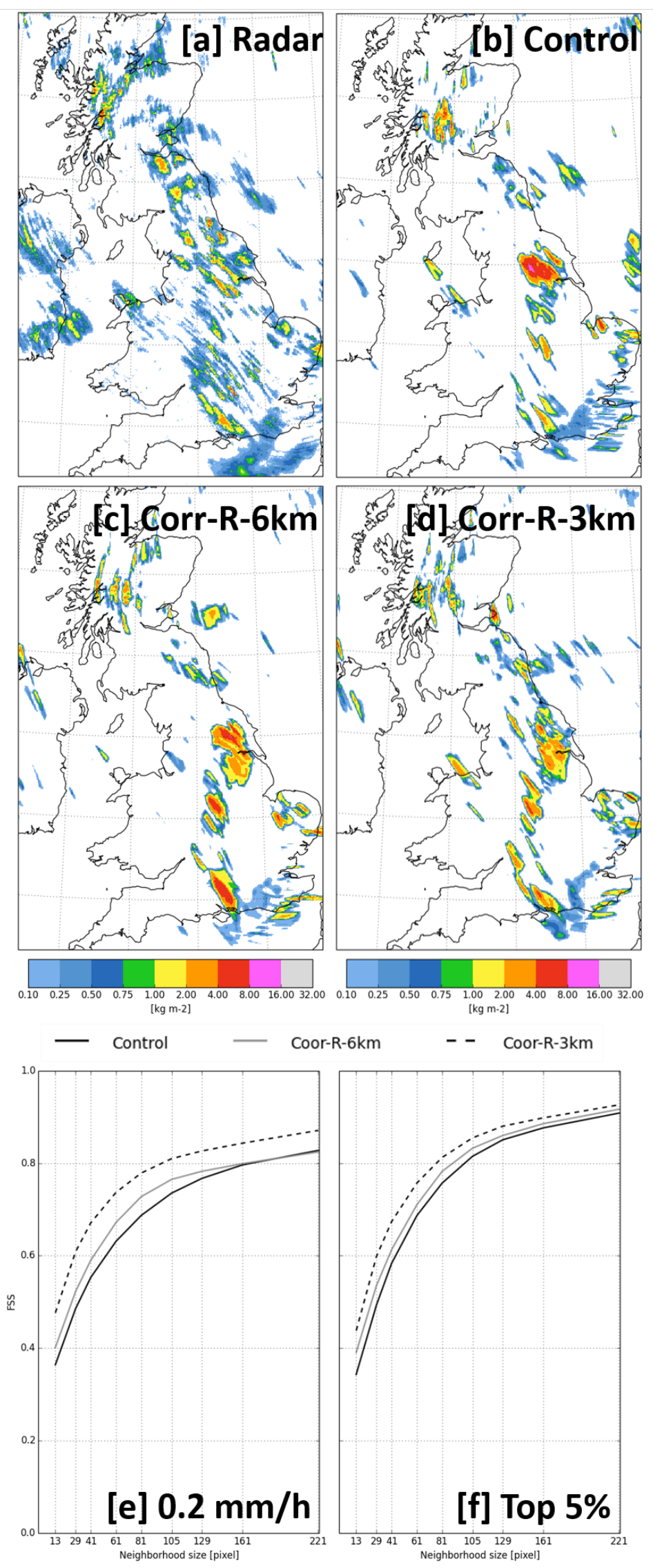

Figure 10. Hourly accumulated precipitation forecasts for 1500 UTC on the 7th of April 2016, for Control [b], Coor-R-6km [c] and Coor-R-3km [d] at T+3. Panel [a] shows the observed radar derived hourly rain accumulation at 1500 UTC. Panels [e] and [f] show the FSS as a function of neighbourhood size for the forecast experiments using thresholds of $0.2 \mathrm{~mm} / \mathrm{h}$ and top $5 \%$ (95th percentile) respectively. 